\title{
LES CERCLES DE REMPLISSAGE DES FONCTIONS MÉROMORPHES OU ENTIËRES ET LE THÉORÈME DE PICARD-BOREL.
}

\author{
Par
}

\author{
HENRI MILLOUX
}

à ŚTRASBOURG.

\section{Introduction.}

Le théorème de M. Picard, relatif à l'indétermination d'une fonction uniforme aux environs d'un point singulier essentiel, est trop classique pour être rappelé ici; il en est de même du théorème de $\mathrm{M}$. Borel sur l'exposant de convergence des zéros de $f(z)-a, f(z)$ étant une fonction entière d'ordre fini.

Ces deux importants théorèmes ont été le point de départ d'un grand nombre de travaux. La théorie des familles normales de fonctions, édifiée par M. Montel, a permis de retrouver certains résultats antérieurs, et de découvrir d'importantes propriétés nouvelles. Parmi celles-ci se trouve le théorème de M. Julia:

Soit $f(z)$ une fonction méromorphe possédant une valeur asymptotique; il existe une suite de points $z(n, f)$ s'éloignant indéfiniment, et jouissant de la propriété suivante: $\varepsilon$ étant un nombre positif arbitrairement petit et $C(n, \varepsilon)$ le cercle de centre $z(n, f)$ et de rayon $\varepsilon|z(n, f)|$, la fonction $f(z)$ prend une infinité de fois toute valeur, sauf deux au plus, dans une suite de cercles $C(n, \varepsilon)$ s'éloignant indéfiniment.

En désignant par $(J)$ une courbe continue allant à l'infini, et en définissant l'argument curviligne d'un point au moyen des courbes $\left(J^{\prime}\right)$ qui se déduisent de $(J)$ par rotation autour de l'origine, il existe une courbe $\left(J^{\prime}\right)$ jouissant de cette 
propriété: dans tout angle curviligne d'ouverture arbitrairement petite, admettant pour bissectrice $\left(J^{\prime}\right)$, se trouvent une suite de points $z(n, f)$ s'éloignant indéfiniment. M. G. Valiron a donné à cette courbe le nom de courbe de Julia.

Dans ma thèse (Journal de Math. 1924), je me suis affranchi de la théorie des familles normales de fonctions. En revenant au théorème de Schottky, et en utilisant une inégalité de $\mathbf{M}$. Carleman, j'ai montré l'existence de ce que j'ai appelé cercles de remplissage, pour une fonction méromorphe $f(z)$ possédant une valeur asymptotique $b$. En faisant la représentation des valeurs de la fonction $f(z)$ sur la sphère de rayon un, les valeurs prises par la fonction dans un cercle de remplissage occupent toute la sphère, sauf peut-être des régions incluses dans deux petits cercles au plus de rayon $e^{-A}$.

La quantité $\frac{I}{A}$, ainsi que le rapport du rayon du cercle de remplissage à la distance de son centre à l'origine, tendent vers zéro lorsque le cercle s'éloigne indéfiniment, et dépendent d'une manière simple de la valeur de la fonction $f(z)$ sur le chemin de détermination $b$ (ou, dans le cas d'une fonction entière, du maximum du module de la fonction sur le cercle $|z|=r$ ).

La question de la recherche du nombre des racines de toute équation $f(z)-a=0$ dans un cercle de remplissage est seulement esquissée.

L'existence des cercles de remplissage et les propriétés de la fonction $f(z)$ dans ces cercles m'ont permis de retrouver et de préciser le théorème de M. Julia et d'autres théorèmes dus à M. Montel.

En 1925, M. Ostrowski a complété le théorème de M. Julia en déterminant les fonctions méromorphes exceptionnelles $J$ qui n'y satisfont pas. Ces fonctions sont très particulières; elles sont d'ordre nul et satisfont en particulier à la condition:

$$
\varlimsup \frac{T(r)}{(\log r)^{2}}<+\infty
$$

$T(r)$ désigne la fonction caractéristique de M. R. Nevanlinna.

Le premier membre de l'inégalité précédente peut d'ailleurs être égal à une constante positive $A$ aussi grande que l'on veut: il existe toujours des fonctions méromorphes exceptionnelles $J$ de $M$. Ostrowski, pours lesquelles:

$$
\overline{\lim } \frac{T(r)}{(\log r)^{2}}=A
$$


Dans plusieurs mémoires, M. G. Valiron a repris l'étude des cercles de remplissage. Il a en particulier réduit le rayon d'un cercle de remplissage d'une fonction entière jusqu'à une valeur qu'un exemple montre minima (à un facteur numérique près); il a établi qu'une fonction méromorphe d'ordre positif possède une suite de cercles de remplissage, et a donné une expression du rayon minimum voisine de la valeur exacte. M. Valiron utilise principalement, dans ses démonstrations, les si remarquables propriétés de la fonction caractéristique $T(r)$ de M. R. Nevanlinna, mises en lumière dans les beaux mémoires de cet auteur.

Enfin, dans un important mémoire publié récemment (Acta math. 1928), M. G. Valiron résont, d'une manière presque complète, la question de la recherche du nombre des zéros de $f(z)-a$ situés dans un cercle de remplissage de la fonction méromorphe $f(z)$. Comme conséquence particulière de ses résultats, il établit l'existence, pour une fonction méromorphe d'ordre positif $\varrho$, d'une direction $D$, qu'il appelle direction de Borel, et qui peut être une direction rectiligne ou curviligne, jouissant de cette propriété: dans tout angle (rectiligne ou curviligne) comprenant $D$ à son intérieur, l'exposant de convergence des zéros de $f(z)-a$ est égal à $\varrho$, sauf pour deux valeurs au plus de $a$.

Une partie de ce présent mémoire apporte un complément à peu près définitif aux récents résultats de $M$. G. Valiron.

La première partie de ce mémoire est consacrée à l'étude des fonctions méromorphes. Certains des résultats saillants de cette première partie sont dus à une collaboration avec M. Valiron.

Dans le chapitre I, en plus des inégalités fondan entales de M. Rolf Nevanlinna, j'utilise, dans l'étude des fonctions méromor ọhes à l'intérieur d'un cercle de rayon fini, un théorème de $\mathbf{P}$. Boutroux relatif au minimum du module d'un polynôme à l'extérieur de couronnes circulaires e rcluant les zéros de ce pólynôme. Deux applications de ce théorème de Boutroux (la deuxième est due à M. Valiron) permettent d'obtenir un énoncé $\mathrm{s}$ mple et général (voir théorème II).

Dans le chapitre II, j'applique les résultats du chajitre I à l'étude de fonctions méromorphes dans tout le plan (ou à l'extérieur d'un cercle); les propriétés obtenues se présentent d'une manière satisfaisante: en ce qui concerne les fonctions méromorphes d'ordre nul, toute fonction $f(z)$ dont le $T(r)$ satisfait à la condition

$$
\overline{\lim } \frac{T(r)}{(\log r)^{2}}=\infty
$$


possède une suite de cercles de remplissage s'éloignant indéfiniment, et, par con. séquent, des coürbes de Julia, ce qui résulte déjà des travaux de M. Ostrowski; mais en plus, ici, nous précisons les valeurs, fonctions de $T(r)$, des rayons des cercles de remplissage, ainsi que le nombre des zéros de $f(z)-a$ situés dans ces cercles.

En ce qui concerne les fonctions méromorphes d'ordre fini, les propriétés obtenues sont également très précises: la valeur minima du rayon d'un cercle de remplissage, valeur donnée en fonction de $T(r)$, est exacte à un facteur numérique près, comme le montre l'exemple des fonctions elliptiques; il en est de même de l'expression du nombre des zéros de $f(z)-a$ situés dans un cercle de remplissage de rayon arbitraire (et, naturellement, supérieure à sa valeur minima).

Des propriétés analogues sont obtenues pour les fonctions méromorphes d'ordre infini.

Enfin, je termine la première partie de ce mémoire en résolvant une question posée par M. Valiron: les valeurs minima des rayons des cercles de remplissage sont différentes dans le cas des fonctions méromorphes et dans le cas des fonctions entières; je montre que la valeur trouvée par $M$. Valiron dans ce dernier cas s'étend à une fonction méromorphe possédant, dans une certaine couronne circulaire, une valeur exceptionelle au sens large du mot; l'énoncé obtenu est particulièrement simple lorsque la fonction est d'ordre fini ou infini.

La deuxième partie est consacrée à l'étude des fonctions entières d'ordre fini.

Dans le chapitre III j'étudie l'extension angulaire des domaines où une fonction entière (d'ordre fini ou non) est supérieure en module à une certaine quantité. J'applique brièvement à l'étude des fonctions entières, d'ordre compris entre $\frac{I}{2}$ et $I$, en montrant qu'un théorème de M. Wiman, relatif aux fonctions entières d'ordre inférieur à $\frac{I}{2}$ (et que l'on retrouve d'ailleurs ici), s'étend à certaines de ces fonctions.

Dans le chapitre IV j'étudie, en utilisant les résultats du chapitre III, la distribution angulaire des cercles de remplissage des fonctions entières d'ordre fini $\varrho$ supérieur à $\frac{\mathrm{I}}{2}$; je montre que, dans certaines couronnes circulaires, il existe au moins deux de ces cercles, tels que les arguments de leurs centres différent au moins d'une quantité voisine de $\frac{\pi}{\varrho}$, ou de $2 \pi-\frac{\pi}{\varrho}$, suivant les cas. Les énoncés sont plus précis lorsqu'il s'agit d'une fonction à croissance très régulière. 
Dans le chapitre $V$, j'étudie plus particulièrement une classe de fonctions prises parmi les fonctions entières d'ordre fini $\varrho$ supérieur à un, ne possédant que deux demi-droites de Borel. Font partie de cette classe toutes les fonctions entières d'ordre fini $\varrho$ supérieur à un ne possédant que deux demi-droites de Julia. M. Valiron avait déjà étendu aux fonctions de cette classe des propriétés que j'avais démontrées pour les fonctions à deux demi-droites de Julia. Je reprends cette étude, et montre en particulier que l'exposant de convergence des zéros de $f(z)-a$ ayant pour argument limite l'un quelconque dés arguments des deux demi-droites de Borel (ces deux arguments ont une différence égale à $\frac{\pi}{\varrho}$ ), est égale à $\varrho$ quel que soit $a$; tandis que l'exposant de convergence d'une autre suite infinie quelconque des zéros de $f(z)-a$ ayant un argument limite différent, est inférieur à $\varrho$ quel que soit $a$.

J'étudie en outre les propriétés de la fonction $f(z)$ dans les deux angles déterminés par les deux demi-droites de Borel.

Les principaux résultats de ce mémoire ont été résumés: dans 3 notes aux Comptes-Rendus en ce qui concerne mes recherches personnelles (19 déc. 1927, 23 janv. et 2 avril 1928), et dans une note aux Compte-Rendus (30 avril 1928) résumant les résultats dus à $\mathrm{M}$. Valiron.

\section{PREMIERE PARTIE.}

\section{Fonctions méromorphes.}

I. Notations employées. - Soit $g(x)$ une fonction méromorphe dans le voisinage du point $x=0$. Nous emploierons les notations suivantes:

$n(r, a)$ désigne le nombre des zéros de $g(x)-a$ situés dans le cercle $|x|=r$.

$$
N(r, a)=\int_{0}^{r}[n(t, a)-n(\mathrm{o}, a)] \frac{d t}{t}+n(\mathrm{o}, a) \log r .
$$

Si $g(0)$ est différent de $a$ (ce sera toujours le cas dans ce mémoire) $n(0, a)$ est nul et l'on a simplement:

$$
N(r, a)=\int_{0}^{r} n(t, a) \frac{d t}{t} .
$$

25-- 2822. Acta mathematica. 62. Imprimé le 17 septembre 1928. 


$$
m(r, a)=\frac{\mathrm{I}}{2 \pi} \int_{0}^{2 \pi} \log \frac{\mathrm{I}}{\left|g\left(r e^{u}\right)-a\right|} d u .
$$

$(\log A$ est égal à $\log A$ si $A>\mathrm{I}$ et à zéro si $A \leq \mathrm{I})$.

$$
\begin{gathered}
m(r, \infty)=\frac{\mathrm{I}}{2 \pi} \int_{0}^{2 \pi}+\log \left|g\left(r e^{i u}\right)\right| d u . \\
T(r, g)=N(r, \infty)+m(r, \infty) .
\end{gathered}
$$

2. - Rappel d'inégalités fondamentales. - C'est M. R. Nevanlinna ${ }^{1}$ qui a défini la fonction caractéristique $T(r, g)$ et a donné les importantes propriétés de cette fonction. Rappelons ici la première inégalité fondamentale:

$$
T(r, g)=m(r, a)+N(r, a)+h(r)
$$

avec:

$$
|h(r)| \leq|\log | c_{\mu}||+\log 2+\log a
$$

$c_{\mu}$ désigne le premier coefficient non nul du développement de la fonction $g(x)-a$ en série de Laurent. Nous nous arrangerons toujours pour que $g(0)$ diffère de $a$; et dans ce cas:

$$
|h(v)| \leq|\log | g(a)-a||+\log 2+\log ^{+} a
$$

La deuxième inégalité fondamentale sera utilisée dans ce mémoire sous différentes formes; sa forme habituelle est la suivante:

$$
T(r, g)<N(r, a)+N(r, b)+N(r, c)+S(r) .
$$

En explicitant les valeurs de certaines constantes entrant dans la fonction $S(r)$, M. Valiron ${ }^{2}$ a donné à cette fonction la forme:

(4) $S\left(r^{\prime}\right)<24 \log T(R, g)+\mathrm{I} 2 \log ^{+} \frac{\mathrm{I}}{R-r}+8 \log ^{+} R+c_{2}(g)+8 \log ^{+} \frac{\mathrm{I}}{|a-b||b-c||c-a|}$

$R$ est un nombre quelconque compris entre $r$ et $2 r$, et l'inégalité (3) est valable dès que $T(r, g)$ dépasse les nombres suivants:

${ }^{1}$ R. Nevanlinna. Zur theorie der meromorphen Funktionen (Acta math. t. 46, 1925).

${ }^{2}$ G. Valiron. Sur la distribution des valeurs des fonctions méromorphes (Acta math., t. 47). 


$$
\begin{aligned}
& c(g) \\
& \log ^{+}|\alpha| \\
& \stackrel{+}{\log } \frac{1}{|\alpha-g(0)|}
\end{aligned}
$$

$\alpha$ désigne l'une quelconque des trois quantités $a, b, c ; c(g)$ et $c_{2}(g)$ sont des constantes dépendant uniquement de la fonction méromorphe $g(x)$. Dans une étude que nous allons bientôt commencer, nous utiliserons des expressions de ces deux constantes $c(g)$ et $c_{2}(g)$, lorsque les trois quantités $a, b, c$ qui figurent dans l'inégalité (3) sont $0, \mathbf{I}, \infty$. Nous utiliserons alors l'inégalité:

$$
\begin{aligned}
T(r, g)<24[N(r, \mathrm{o})+N(r, \mathrm{I})+N(r, \infty)]+36 \log \frac{\mathrm{I}}{\mathrm{I}-\frac{r}{R}}+ \\
+\mathrm{I} 2 \log ^{+}|g(0)|+\log ^{+} \frac{\mathrm{I}}{R\left|g^{\prime}(\mathrm{o})\right|}+C .
\end{aligned}
$$

$C$ est une constante numérique. La forme précise de cette inégalité résulte des calculs récents de M. Valiron. ${ }^{1}$

3. - Rappel d'un théorème fondamental de P. Boutroux. - Ce théorème est le suivant:

Etant donnés $m$ points $P_{1}, P_{2}, \ldots P_{m}$ situés sur un segment de largeur un, et $Q$ un point situé sur le même segment, l'inégalité:

$$
P_{1} Q . P_{2} Q \ldots P_{m} Q>k^{m}
$$

est vérifiée, sauf pour des points $Q$ compris dans des intervalles de largeur totale $4 e k^{2}$

Bien entendu, nous choisirons constamment $k$ de façon que cette longeur totale soit inférieure à un, de sorte que l'inégalité (6) sera vérifiée au moins pour une position du point $Q$.

${ }^{1} \mathrm{G}$. Valiron. Recherches sur le théorème de M. Borel dans la théorie des fonctions méro. morphes, (Acta math. t. 52, p. 72).

${ }^{2}$ P. Boutroux. Sur quelques propriétés des fonctions entières, thèse (Acta math. 1903). Le théorème de P. Boutroux fixe comme longueur totale des intervalles la quantité $a k$, $a$ etant une constante numérique supérieure à $4 e$; récemment, $\mathrm{M}$. André Bloch a énoncé sans démonstration un théorème plus précis que le théorème de Boutronx; puis M. Henri Cartan a précisé ce dernier énoncé de M. André Bloch dans un théorème publié dans les Compte-Rendus (t. I85, I928, p. 624). Nous avons pris comme longueur totale des intervalles celle qui résulte du théoreme de M. H. Cartan, dans le but de diminuer certaines constantes numériques. 


\section{CHAPITRE I.}

\section{Fonctions méromorphès dans un cercle.}

4. Soit $g(x)$ une fonction méromorphe dans un certain voisinage de l'origine, et soit $Q$ un point d'affixe $x_{0}$, en lequel la fonction est différente de $a$. Si l'on désigne par $P_{1}, P_{2}, \ldots P_{m}$ les points intérieurs au cercle de centre $x_{0}$ et de rayon $R$, en lesquels la fonction $g(x)$ est égale à $a$ (chacun de ces points étant pris avec son ordre de multiplicité), on $\mathbf{a}$ :

$$
N\left(\left|x-x_{0}\right|=R, a\right)=\log \frac{R^{m}}{P_{1} Q . P_{2} Q \ldots P_{m} Q}
$$

On obtient une limite supérieure du deuxième membre de cette égalité en alignant les points $Q$ et $P_{1}, P_{2}, \ldots P_{m}$ sur le même segment de largeur $R$, et, d'après le théorème de Boutroux, il existe des points $Q$ vérifiant l'inégalité:

$$
\frac{P_{1} Q \cdot P_{2} Q \ldots P_{m} Q}{R^{m}}>\underset{\mathrm{I} 2 \mathrm{O}^{m i}}{\mathrm{I}}
$$

dans tout intervalle de largeur $\frac{R}{10}$.

En retournant à des points $P_{1}, P_{2}, \ldots P_{m}$ disposés d'une façon quelconque dans le cercle $|x|=$ I par exemple, chaque couronne circulaire de centre $A$ et d'épaisseur $\frac{I}{\mathrm{I} 2}$ contient nécessairement une circonférence de centre $A$ en tout point $Q$ de laquelle on a l'inégalité:

$$
P_{1} Q . P_{2} Q \ldots P_{m} Q>k^{m} \quad\left(k=\frac{\mathrm{I}}{\mathrm{I} 2 \mathrm{O}}\right)
$$

de même, si on considère des cordes parallèles à une direction fixe, sur tout inter-

valle de largeur $\frac{\mathrm{I}}{\mathrm{IO}}$ (comptée perpendiculairement à la direction des cordes), il existe nécessairement une corde sur laquelle l'inégalité précédente est vérifiée. Nous appellerons dans la suite corde ou cercle de Boutroux une corde ou cercle sur lequel cette inégalité est vérifiée.

Ceci posé, soient $P_{1}, P_{2}, \ldots P_{m}$ les zéros de la fonction méromorphe $g(x)$ dans le cercle $|x|=\mathrm{I} ; P_{1}^{\prime}, P_{2}^{\prime}, \ldots P_{m^{\prime}}^{\prime}$, les zéros de $g(x)-\mathrm{I} ; P_{1}^{\prime \prime}, P_{2}^{\prime \prime}, \ldots P_{m^{\prime \prime}}^{\prime \prime}$ les zéros de $\frac{\mathrm{I}}{g(x)}$, dans le même cercle. 
Nous aurons des cordes et des cercles de Boutroux, relatifs à la fois aux zéros de $g(x), g(x)-\mathrm{I}, \frac{\mathrm{I}}{g(x)}$, dans tout intervalle linéaire (cordes parallèles à une direction fixe) ou circulaire (cercles intérieurs à une couronne circulaire et concentriques) de largeur $\frac{\mathrm{I}}{10}$ si nous prenons $\frac{\mathrm{I}}{360}$ pour valeur de $k$. Fixons de suite la position de la corde et du cercle de Boutroux choisis pour la fonction:

La distance du point $O$ à la corde est inférieure à $\frac{\mathrm{I}}{2 \mathrm{O}}$.

Le cercle, appelé $(\Gamma)$, a son centre en $O^{\prime}$, projection du point $O$ sur la corde précédente. Son rayon est compris entre $\frac{\mathrm{I}}{9}$ et $\frac{\mathrm{I}}{9}+\frac{\mathrm{I}}{\mathrm{IO}}$, de sorte que la distance de ses points au cercle $|x|=\mathrm{I}$ est supérieure à $\frac{\mathrm{I} 23}{\mathrm{I} 8 \mathrm{O}}$.

Nous désignons par $A$ et $B$ les points d'intersection du cercle et de la corde de Boutroux.

Soit $x_{0}$ un point quelconque de la corde $A B$ et du cercle $(\Gamma)$. D'après l'inégalité de Boutroux, on a:

$$
N\left(\left|x-x_{0}\right|=R, a\right)<6 n(|x|=\mathrm{I}, a) .
$$

$a$ désigne l'une des quantités $\mathrm{O}, \mathrm{I}, \infty ; R$ est le rayon du cercle de centre $x_{0}$ tangent au cercle $|x|=\mathrm{I}$.

Supposons les nombres $m, m^{\prime}, m^{\prime \prime}$ des zéros de $g(x), g(x)-\mathrm{I}, \frac{\mathrm{I}}{g(x)}$ situés dans le cercle $|x|=\mathrm{I}$, inférieurs à $n$. D'après ce qui précède, on a:

$$
N\left(\left|x-x_{0}\right|=R, \mathrm{o}\right)+N\left(\left|x-x_{0}\right|=R, \mathrm{I}\right)+N\left(\left|x-x_{0}\right|=R, \infty\right)<\mathrm{I} 8 n
$$

et par suite, d'après l'inégalité (5), la fonction caractéristique vérifie l'inégalité:

(7) $T\left(\left|x-x_{0}\right|=\frac{\mathrm{I} 22}{\mathrm{I} 23} R, g\right)<432 n+\mathrm{I} 2 \stackrel{+}{\log }\left|g\left(x_{0}\right)\right|+4 \stackrel{+}{+} \frac{\mathrm{I}}{\left|g^{\prime}\left(x_{0}\right)\right|}+C$.

Proposons-nous de rechercher une limite supérieure du nombre des zéros de $g(x)-a$ dans un certain cercle concentrique au cercle $|x|=\mathrm{I}$. La fonction $g(x)$ ne prend pas plus de $n$ fois, dans le cercle $|x|=\mathrm{I}$, chacune des valeurs $0, \mathrm{I}, \infty$. Il en est de même des cinq fonctions méromorphes que l'on déduit de $g(x)$ par les transformations homographiques bien connues. Remarquons en passant que 
ces transformations laissent invariantes les distances sphériques de deux valeurs quelconques de $g(x)$. Quitte à effectuer l'une de ces transformations, nous pourrons done supposer que l'on a:

$$
\begin{gathered}
\frac{\mathrm{I}}{2} \leq\left|g\left(O^{\prime}\right)\right| \leq 2 \\
|a|<\mathrm{I}
\end{gathered}
$$

L'inégalité (7) n'est appliquable que si $g^{\prime}\left(x_{0}\right)$ n'est pas petit; il est donc nécessaire, dans cette étude, de distinguer deux cas.

Premier cas. - En un point $x_{0}$ de la corde $A B$ ou du cercle $(\Gamma)$, on a:

$$
\log \left|g^{\prime}\left(x_{0}\right)\right|>-n \text {. }
$$

Nous choisirons pour $x_{0}$ le premier point satisfaisant à l'inégalité précédente, rencontré en cheminant à partir du point $O^{\prime}$ de sorte que $g\left(x_{0}\right)$ est inférieur à 3 des que $n$ est supérieur ou égal à 3 , et par suite, d'après l'inégalité (7):

$$
T\left(\left|x-x_{0}\right|=\frac{122}{123} R, g\right)<436 n+C^{\prime} .
$$

$C^{\prime}$ étant une constante numérique.

Appliquons maintenant la première inégalité fondamentale de M. R. Nevanlinna, rappelée au $\mathrm{n}^{\circ}$ :

$$
\begin{gathered}
N\left(\left|x-x_{0}\right|=\frac{\mathrm{I} 22}{\mathrm{I} 23} R, a\right)+m\left(\left|x-x_{0}\right|=\frac{\mathrm{I} 22}{\mathrm{I} 23} R, a\right)=T\left(\left|x-x_{0}\right|=\frac{\mathrm{I} 22}{\mathrm{I} 23} R, g\right) \\
+h\left(\left|x-x_{0}\right|=\frac{\mathrm{I} 22}{\mathrm{I} 23} R\right) .
\end{gathered}
$$

Or:

$$
\left|h\left(\left|x-x_{0}\right|=\frac{\mathrm{I} 22}{\mathrm{I} 23} R\right)\right| \leq|\log | g\left(x_{0}\right)-a||+\log 2+\stackrel{+}{+} a
$$

et comme le modưle de $a$ est inférieur à un, on en déduit:

$$
N\left(\left|x-x_{0}\right|=\frac{\mathrm{I} 22}{\mathrm{I} 23} R, a\right)<436 n+C^{\prime \prime}+\log ^{+} \frac{\mathrm{I}}{\left|g\left(x_{0}\right)-a\right|} .
$$

Le cercle $\left|x-x_{0}\right|=\frac{\mathrm{I} 22}{\mathrm{I} 23} R . \frac{63}{\mathrm{I} 22}$ contient done un nombre de zéros de $g(x)-a$ qu'il est facile de déduire de la limitation précédente de $N$, d'après l'expression 
de $N(r, a)$ en fonction de $n(r, a)$; d'ailleurs ce cercle contient à son intérieur le cercle $|x|=\frac{I}{20}$. Nous pouvons énoncer le résultat suivant:

A l'intérieur de la circonférence $|x|=\frac{1}{20}$, le nombre des zéros de $g(x)-$ a est inférieur $\grave{a}$

$$
660 n+C+2 \stackrel{+}{\log } \frac{\mathrm{I}}{|A-a|}
$$

$C$ est une constante numérique; $A$ est un nombre complexe fixe.

Deuxième cas. - En tout point du cercle $(\Gamma)$ et de la corde $A B$, on $a$ :

$$
\log \left|g^{\prime}(x)\right| \dot{<}<-n
$$

et par suite:

$$
|g(x)|<3
$$

(On suppose $n \geq 3$ ).

Désignons par $x_{2}$ l'affixe du centre $O^{\prime}$ du cercle $(\Gamma)$. Dans ce cercle on a:

$$
N\left(\left|x-x_{2}\right|, \infty\right)<6 n
$$

et sur la circonférence $(\Gamma)$, la fonction:

$$
m\left(\left|x-x_{2}\right|, \infty\right)=\frac{\mathrm{I}}{2 \pi} \int_{0}^{2 \pi}+\log \left|g\left(\left|x-x_{2}\right| e^{i u}\right)\right| d u
$$

est bornée, puisque le module de $g(x)$ sur ce cercle est inférieur à 3 .

Il en résulte qu'à l'intérieur du cercle $(\Gamma)$, on a l'inégalité:

$$
T\left(\left|x-x_{2}\right|, g\right)<6 n+2 \text {. }
$$

La première inégalité de M. R. Nevanlinna, utilisée comme plus haut, fournit une limite supérieure du nombre des zéros de $g(x)-a$ dans un cercle concentrique à $(\Gamma)$ et de rayon moindre. On choisit ce rayon de manière que ce dernier cercle contienne à son intérieur le cercle $|x|=\frac{1}{20}$, et on obtient aisément, commme limitation supérieure du nombre des zéros de $g(x)-a$ dans ce cercle:

$$
C+\text { I I } \stackrel{+}{\log } \frac{\mathrm{I}}{|A-a|}+66 n
$$


En résumé, dans les deux cas, le nombre des zéros de $g(x)$ - $a$ intérieurs au cercle $|x|=\frac{I}{20}$ est inférieur à

$$
660 n+C+\mathrm{II} \stackrel{+}{\log } \frac{\mathrm{I}}{|A-a|}
$$

$A$ étant un nombre complexe fixe, et $C$ une constante numérique.

Rappelons que nous avons choisi, entre 6 fonctions, la fonction $g(x)$ de façon que $a$ soit inférieur en module à un ( $A$ peut être également supposé limité en module, par deux par exemple). Revenons maintenant à l'une des 5 fonctions déduites de $g(x)^{\circ}$ par l'une des transformations homographiques bien connues laissant invariantes les quantités $0,1, \infty$. Il suffira, dans la limitation précédente, de remplacer $|A-a|$ par son équivalent après la transformation, c'est-à-dire, à un facteur près (compris entre deux constantes numériques), la distance sphérique des deux valeurs transformées de $a$ et $A$, d'où le théorème suivant:

5. Soit $g(x)$ une fonction méromorphe dans le cercle $|x|=\mathrm{I}$, et ne prenant pas plus de $n$ fois chacune des valeurs $\mathrm{O}, \mathrm{I}, \infty$. Dès que $n$ dépasse une certaine constante numérique, le nombre des racines de l'équation $g(x)-a=0$ intérieures au cercle $|x|=\frac{\mathbf{I}}{20}$ est inférieur $\grave{a}$

$$
665 n+11+\frac{1}{\log } \frac{1}{d}
$$

d désignant la distance sphérique de a à une certaine valeur exceptionnelle possible $A$.

L'exemple de la fonction $g(x)=3+x^{p}, p$ étant un entier positif, montre que cette valeur exceptionnelle $A$ peut exister (Ici $A=3 ; n=0$ ).

6. Passous maintenant à l'étude d'une fonction méromorphe $f(x)$ ne prenant pas plus de $n$ fois trois valeurs $\alpha, \beta, \gamma$ dont les distances sphériques prises deux à deux sont supérieures à $\delta$. La transformation:

$$
g(x)=\frac{f(x)-\alpha}{f(x)-\beta}: \frac{\gamma-\alpha}{\gamma-\beta}
$$

fait correspondre, à une telle fonction, une fonction $g(x)$ du type étudié au $n^{\circ} 4$.

L'application $d u$ théorème du $n^{\circ} 5$ nécessite une étude sommaire des correspondances entre les distances sphériques dans la transformation (8).

Les transformations élémentaires: 


$$
F=\frac{\mathrm{I}}{f} ; \quad F=f-a ; \quad F=k f
$$

où le module de $a$ est inférieur à un, et où $k$ est un nombre positif compris entre $\frac{1}{3}$ et 3 , multiplient la distance sphérique de deux valeurs quelconques de $f$ par une quantité comprise entre deux constantes numériques positives. On peut effectuer sur $f(x)$ une combinaison de ces transformations de manière à annuler l'une des trois quantités $\alpha, \beta, \gamma$ et à prendre les deux autres inférieures en module à un. Il est loisible de choisir la quantité nulle (soit, par exemple, $\alpha$ ) de façon que l'on ait:

$$
\begin{gathered}
|\beta| \leq|\gamma| \\
|\gamma-\beta| \geq|\beta| .
\end{gathered}
$$

Le module de $\beta$ est ainsi supérieur à $k \delta, k$ étant une constante numérique. La transformation: $F=\frac{f}{\beta}$ permet de passer de $\alpha=0, \beta, \gamma$, aux trois quantités $\alpha^{\prime}=\dot{0}, \dot{\beta}^{\prime}=\mathrm{I}$, et $\gamma^{\prime}$, cette dernière quantité vérifiant les inégalités: $\left|\gamma^{\prime}\right| \geq \mathrm{I}$, et $\left|\gamma^{\prime}-\mathrm{I}\right| \geq \mathrm{I}$.

Cette transformation multiplie les distances sphériques de deux valeurs quelconques de $f$ par une quantité qui est supérieure à $k^{\prime} \delta, k^{\prime}$ désignant une constante numérique.

Enfin, une combinaison des premières transformations élémentaires permet de passer des trois quantités $\alpha^{\prime}, \beta^{\prime}, \gamma^{\prime}$ aux trois quantités: $\alpha^{\prime \prime}=\infty, \beta^{\prime \prime}=\mathrm{I}, \gamma^{\prime \prime}=0$.

L'ensemble de toutes ces transformations multiplie la distance sphérique de deux valeurs quelconques de $f$ par une quantité supérieure à $k \delta, k$ désignant une constante numérique.

7. Revenons à la fonction $g(x)$. Celle-ci ne prend pas plus de $n$ fois les valeurs $\mathrm{O}, \mathrm{I}, \infty$. D'après le théorème du $\mathrm{n}^{\circ} 5$, le nombre des zéros de $g(x)-a^{\prime}$, intérieur au cercle $|x|=\frac{\mathrm{I}}{20}$, est inférieur à

$$
665 n+\mathrm{II} \log \frac{\mathrm{I}}{d^{\prime}}
$$

$d^{\prime}$ désignant la distance sphérique de $a^{\prime}$ à une certaine valeur exceptionnelle possible $A^{\prime}$. Les valeurs $a^{\prime}$ et $A^{\prime}$ de $g(x)$ correspondent aux. valeurs $a$ et $A$ de $f(x)$, dont la distance sphérique $d$ satisfait, d'après l'étude $d u n^{\circ} \sigma$, à l'inégalité:

26 - 2822. Acta mathematica. 52. Imprimé le 17 septembre 1928. 


$$
d<\frac{d^{\prime}}{k \delta}
$$

d'où le théorème suivant:

I. Soit $g(x)$ une fonction méromorphe dans le cercle $|x|=\mathrm{I}$, et n'y prenant pas plus de $n$ fois trois valeurs $\alpha, \beta, \gamma$ dont les distances sphériques sont supérieures $\grave{a} \delta$. Dès que $n$ dépasse une constante numérique, le nombre des zéros de $f(x)-a$ intérieurs au cercle $|x|=\frac{\mathrm{I}}{2 \mathrm{O}}$ est inférieur $\grave{a}$ :

$$
670 n+\mathrm{II} \log \frac{\mathrm{I}}{d}+\mathrm{II} \log \frac{\mathrm{I}}{\delta}
$$

$d$ désignant la distance sphérique de a à une certaine valeur exceptionnelle possible $A$.

8. Ce théorème admet immédiatement le corollaire snivant:

Corollaire. Soit $f(x)$ une fonction méromorphe dans un cercle $(C)$. Si dans le cercle concentrique $\grave{a}(C)$ et de rayon 20 fois moindre, la fonction $f(x)$ prend plus de $n$ fois deux valeurs de distance sphérique supérieure à $e^{-n}$, cette fonctión prend, dans le cercle $(C)$, toute valeur, un nombre de fois supérieur $\grave{a} \frac{n}{700}$, sauf peut-être des valeurs dont les représentations sphériques sont incluses dans deux petits cercles de rayon $e^{-n .1}$

Ce corollaire nous sera utile dans la suite. C'est en somme une application particulière $d u$ théorème $I$, dans lequel on fait: $d=e^{-n}$. $^{2}$

9. Soit $f(z)$ une fonction méromorphe dans une certaine région du plan, contenant à son interieur un domaine $(D)$ dans lequel elle prend $N$ fois au moins toute valeur $\alpha$ dont la représentation sphérique est intérieure à un cercle de rayon fini; pour fixer les idées, nous supposerons par exemple que $\alpha$ désigne une valeur quelconque prise parmi les valeurs de modules inférieurs à $\frac{I}{2}$.

Partageons le domaine $D$ en $p$ domaines partiels $D_{1}, D_{2}, \ldots D_{p}$, et construi-

1 Au lieu de prendre un cercle concentrique à $(C)$ et de rayon 20 fois moindre, on peut prendre un cercle dont le rayon est égal au produit de $(I-\varepsilon)$ par le rayon du cercle $(C)$. C'est ce qu'a fait M. Valiron (Compte-Rendus, 2 avril I928, p. 935) par représentation conforme.

${ }^{2}$ C'est la valeur que j'avais prise pour $d$. Jobtenais des résultats identiques, dans la plupart des cas, à ceux que nous allons obtenir avec la méthode de M. Valiron, qui montre l'importance de la conservation du terme i I $\log \frac{\mathrm{I}}{d}$ figurant dans le théorème $\mathrm{I}$. Ce résultat de $\mathrm{M}$. Valiron, que nous allons développer ici, est résumé dans une note des Compte-Rendus (30 avril I928) 
sons les cercles $\left(C_{1}\right),\left(C_{2}\right), \ldots\left(C_{p}\right)$ concentriques aux plus petits cercles contenant les domaines partiels, et de rayons 20 fois plus grands. Supposons que dans chacun de ces cercles le nombre des zéros de $f(z)-a$ soit inférieur à $n$, pour trois valeurs $a$ dont les distances sphériques, prises deux à deux, sont supérieures à $\delta$.

Il résulte du théorème $\mathrm{I}$ que dans un domaine partiel $\left(D_{i}\right)$ quelconque, le nombre des zéros de $f(z)-\alpha$ est inférieur à:

$$
670 n+\text { I I } \log \frac{\mathrm{I}}{\delta}+\text { I I } \stackrel{+}{+} \frac{\mathrm{I}}{\left|\alpha-A_{i}\right|} .
$$

$A_{i}$ désigne une certaine valeur exceptionnelle possible, pouvant d'ailleurs varier avec le domaine $\left(D_{i}\right)$ choisi.

Le nombre total des zéros de $f(z)-a$ dans l'ensemble des domaines $\left(D_{i}\right)$, c'est-à-dire dans le domaine $(D)$, est donc inférieur à:

$$
670 n p+\text { I I } p \log \frac{\mathrm{I}}{\delta}+\mathrm{I} \text { I } \stackrel{+}{\log } \frac{\mathrm{I}}{\left|\alpha-A_{1}\right| \cdots\left|\alpha-A_{p}\right|} .
$$

M. Valiron ${ }^{1}$ a eu l'idée d'appliquer une seconde fois le théorème de $P$. Boutroux sous la forme suivante: puisque nous pouvons prendre pour $\alpha$ une valeur quelconque (inférieure en module à $\frac{\mathrm{I}}{2}$ ) choisissons-la telle que:

$$
\left|\alpha-A_{1}\right| \cdots\left|\alpha-A_{p}\right|>\frac{\mathrm{I}}{\mathrm{I} 2^{p}} \text {. }
$$

Il existe d'après le théorème de Boutroux, de telles valeurs $\alpha$ (et même une infinité). Pour ces valeurs, le nombre des zéros de $f(z)-\alpha$ situés dans le domaine $D$ est inférieur à:

$$
670 n p+\text { I I } p \log \frac{\mathrm{I}}{\delta}+33 p
$$

Il y a contradiction avec l'hypothèse si l'on prend $\delta=e^{-n}$ et $700 n p=N$. D'où le théorème suivant, d'énoncé un peu plus général:

II. Soit $f(z)$ une fonction méromorphe dans une certaine région du plan, contenant à son intérieur un domaine $(D)$ dans lequel elle prend plus de $N$ fois toute valeur dont la représentation sphérique est incluse dans un cercle de rayon $\frac{\mathrm{I}}{2}$. On

1 Voir sa note des Compte-Rendus, citée dans la note précédente. 
partage le domaine $(D)$ en $p$ domaines partiels et on construit les cercles $\left(C_{1}\right),\left(C_{2}\right), \ldots$ $\left(C_{p}\right)$ concentriques aux cercles contenant les domaines partiels, et de rayons 20 fois plus grands. ${ }^{1}$ Ceci posé, il existe nécessairement un cercle $\left(C_{i}\right)$ à l'intérieur duquel la fonction méromorphe $f(z)$ prend plus de $\frac{N}{700 p}$ fois toute valewr, sauf peut-être des valeurs dont les représentations sphériques sont incluses dans deux petits cercles de rayons $e^{-\frac{N}{700 p}}$.

Ce théorème est applicable dès que $\frac{N}{700 p}$ dépasse une certaine constante numérique.

Il est encore vrai si la fonction $f(z)$ prend, dans le domaine $(D)$, plus de $N$ fois tonte valeur dont la représentation sphérique est incluse dans un cercle de rayon $r$ au lieu de $\frac{\mathrm{I}}{2}$; mais à condition que $\frac{\mathrm{I}}{r}$ ne soit pas trop grand par rapport à $\frac{N}{700 p}$ (la valeur de $\frac{I}{r}$ est limitée par une fonction de $\frac{N}{700 p}$ tendant vers l'infini avec $\frac{N}{700 p}$; la forme exacte de cette fonction se déduit aisément de l'application du théorème de Boutroux à ce cas un peu plus général).

\section{CHAPITRE II.}

\section{Fonctions méromorphes dans le plan.}

Io. Nous allons passer maintenant à l'étude des fonctions méromorphes dans tout le plan. Les résultats que nous allons obtenir sont aussi valables pour des fonctions méromorphes à l'extérieur d'un cercle, et, par suite, sont applicables à l'étude d'une fonction analytique aux environs d'un point singulier essentiel isolé.

I I. Soit $f(z)$ une fonction méromorphe dans tout le plan, et dont la fonction caractéristique est désignée par $T(r)$. Nous appliquerons le théorème II à cette fonction, en choisissant pour domaine $(D)$ la couronne circulaire $(C)$ définie par les inégalités:

$$
r<|z|<R
$$

${ }^{1}$ Ces cercles doivent être, bien entendu, intérieurs à la région dans laquelle la fonction $f(z)$ est méromorphe. 
Quitte à remplacer $f(z)$ par $\frac{\mathrm{I}}{f(z)}$, et à déplacer l'origine, nous supposerons:

$$
\begin{aligned}
& |f(\mathrm{o})| \leq \mathrm{I} \\
& \left|f^{\prime}(\mathrm{o})\right| \neq \mathrm{o} .
\end{aligned}
$$

Pour déterminer une limite inférieure $N$ du nombre des zéros de $f(z)-a$ situés dans la couronne circulaire $(C)$, et caractériser les valeurs de a pour lesquelles cette limite est valable, nous utiliserons les deux inégalités fondamentales de M. R. Nevanlinna.

I2. Commençons par l'étude des fonctions méromorphes d'ordre nul ou d'ordre fini. La fonction caractéristique vérifie alors l'inégalité:

$$
\log T(R)<k \log R
$$

$k$ étant une constante positive.

Il résulte alors des inégalités (3) et (4) qu'il existe nécessairement une certaine valeur $\alpha_{0}$, dont la distance à $f(0)$ est comprise entre $\frac{3}{2}$ et 2 , et telle que pour toutes les valeurs de a satisfaisant à l'inégalité:

on a l'inégalité:

$$
\left|a-\alpha_{0}\right|<\mathrm{I}
$$

$$
N(r, a)>\frac{\mathrm{I}}{4} T(r)
$$

Wn effet, s'il n'existait pas de telle valeur $\alpha_{0}$, on pourrait trouver trois valeurs $a, b, c$ mettant en défaut l'inégalité fondamentale (3).

D'ailleurs, pour de telles valeurs $a$, d'après les inégalités (I) et (2), on a:

$$
N(r, a)<T(r)+C
$$

$C$ désignant une constante absolue.

Passons de la fonction $N(r, a)$ à la fonction $n(r, a)$ :

$$
N(k r, a)=\int_{0}^{k r} n(t, a) \frac{d t}{t}>n(r, a) \log k
$$


et:

$$
N(R, a)=N(r, a)+\int_{r}^{R} n(t, a) \frac{d t}{t}<N(r, a)+n(R, a) \log \frac{R}{r}
$$

D'après ces deux inégalités, le nombre des zéros de $f(z)-a$ situés dans la couronne $(C)$ est supérieur à:

$$
\frac{N(R, a)-N(r, a)}{\log \frac{R}{r}}-\frac{N(k r, a)}{\log k}
$$

D'où la proposition suivante:

III. Soit $f(z)$ une fonction méromorphe d'ordre nul ou fini, à fonction caractéristique $T(r)$. Dans la couronne circulaire $(C)$ définie par les inégalités:

$$
r<|z|<R
$$

le nombre des zéros de $f(z)-a$ est supérieur $\grave{a}$ :

$$
\frac{T(R)}{\text { I } 5 \log \frac{R}{r}}
$$

pour toutes les valeurs de a dont les représentations sphériques sont incluses dans un cercle de rayon $\frac{\mathrm{I}}{2}$, et ceci dès que $T(R)$ dépasse les trois quantités suivantes:

(9)

$$
\left\{\begin{array}{l}
c(f) \\
\text { I2 } T(r) \\
\text { I2 } \frac{T(k r)}{\log k} \log \frac{R}{r} .
\end{array}\right.
$$

$c(f)$ est une constante dépendant uniquement de la fonction méromorphe $f(z)$; $l$ est un nombre positif quelconque compris entre I et $\frac{R}{r}$. On pourra, par, exemple, prendre $k r=\sqrt{R r}$ et remplacer les deux dernières quantités par la quantité unique: $24 T(\sqrt{R r})$.

13. La proposition précédente nous fixe une limite inférieure du nombre des zéros de $f(z)-a$ dans la couronne circulaire $(C)$. Opérons maintenant le 
partage de cette couronne en $p$ domaines partiels, par $q$ demi-droites issues de $o$, faisant entre elles des anglẹs égaux à $\frac{2 \pi}{q}$, et par des cercles de centre $o$, relativement équidistants, de façon à constituer $p$ quadrilatères curvilignes semblables au quadrilatère curviligne défini par les inégalités:

$$
\begin{gathered}
\mathrm{I}<|z|<\mathrm{I}+\frac{2 \pi}{q} \\
-\frac{\pi}{q}<\operatorname{Arg} z<+\frac{\pi}{q} .
\end{gathered}
$$

Le nombre $p$ est alors égal à

$$
\frac{q \log \frac{R}{r}}{\log \left(1+\frac{2 \pi}{q}\right)} .
$$

L'application du théorème II fournit le théorème suivant:

IV. Soit $f(z)$ une fonetion méromorphe d'ordre nul ou fini à fonction caractéristique $T(r)$. Dans la couronne circulaire $(C)$ définie par les inégalités:

$$
r<|z|<R
$$

se trouve un point, de module $r_{i}$, centre d'un cercle de remplissage $\left(C_{i}\right)$, de rayon $\frac{\mathrm{IOO} r_{i}}{q}$, à l'intérieur duquel la fonction $f(z)$ prend plus de

$$
n_{1}=\frac{1}{900 q^{2}} \cdot \frac{T(R)}{\left(\log \frac{R}{r}\right)^{2}}
$$

fois toute valeur, sauf peut-être des valeurs dont les représentations sphériques sont incluses dans deux petits cercles de rayon $e^{-n_{1}}$.

Les conditions d'application de ce théorème sont les suivantes:

${ }^{\circ}{ }^{\circ}$. $n_{1}$ et $q$ doivent dépasser des constantes numériques (et par suite, $n_{1}$ et $q$ sont aussi limités supérieurement par des fonctions de $T(r)$ ).

$2^{\circ}$. $T(R)$ doit être supérieur aux trois quantités (9).

14. L'application de ce théorème aux fonctions méromorphes d'ordre nul conduit immédiatement au théorème suivant: 
V. La fonction méromorphe $f(z)$ d'ordre nul possède des cercles de remplissage uus de l'origine sous des angles tendant vers zéro lorsque les distances à l'origine des centres de ces cercles tendent vers l'infini, lorsque la fonction caractéristique $T(r)$ satisfait $\grave{a}$ la condition:

$$
\varlimsup \frac{T(R)}{(\log R)^{2}}=\infty
$$

Dans une suite quelconque de cercles de remplissage s'éloignant indéfiniment, la fonction $f(z)$ prend une infinité de fois toute valeur, sauf deux au plus, et toute courbe passant par les centres de ces cercles est une courbe de Julia.

Le théorème $\mathrm{V}$ est aussi une conséquence de résultats antérieurs dus à $\mathbf{M}$. Ostrowski. ${ }^{1}$ M. Ostrowski a déterminé les fonctions méromorphes exceptionnelles $J$, e'cst-à-dire les fonctions ne possédant pas de courbe de $J$ ulia. 11 a montré que la fonction caractéristique $T(v)$ d'une telle fonction méromorphe vérifie l'égalité:

$$
\varlimsup \frac{T(R)}{(\log R)^{9}}=A
$$

$A$ est une constante positive ou nulle: il a donné des exemples de ces fonctions, pour lesquelles $A$ a une valeur donnée à l'avance (On peut prendre une fonction méromorphe à l'extérieur d'un cercle, et invariante par la substitution $(r ; s r)$ ).

I5. Le théorème IV s'applique à toutes les fonctions méromorphes d'ordre nul vérifiant l'inégalité:

$$
\varlimsup \frac{T(R)}{(\log R)^{2}}>0
$$

(sans l'égalité).

Dans le cas de fonctions méromorphes pour lesquelles:

$$
\varlimsup \frac{T(R)}{(\log R)^{2}}=\infty
$$

il fournit, en outre du théorème $V$, des renseignements quantitatifs intéressants sur la valeur minima du rayon d'un cercle de remplissage, et sur le nombre des racines des équations $f(z)-a=0$, intérieures aux cercles de remplissage.

\footnotetext{
${ }^{1}$ A. Ostrowski. Uber Folgen analytischer Functionen... (Math. Zeitschrift, Bd 24, 1925, p. 24I).
} 
I6. Dans le cas des fonctions méromorphes d'ordre nul, l'épaisseur relative de la couronne circulaire $(C)$, dans laquelle se trouve le cercle de remplissage $\left(C_{i}\right)$, peut tendre vers l'infini avec $r$. Dans le cas d'une fonction méromorphe d'ordre fini $\varrho$, on peut établir aisément, comme l'a fait M. Valiron, l'existence d'une suite de couronnes circulaires $(C)$ d'épaisseur relative finie.

En effet, en choisissant $R=k^{2} r$, d'après la deuxième condition d'application du théorème IV, $T\left(k^{2} r\right)$ doit être supérieur à " $c(f)$ et à $24 T(k r)$.

On ne peut avoir constamment:

$$
T\left(k^{2} r\right)<24 T(k r)
$$

sans avoir:

$$
T(R)<A \cdot R^{\frac{\log 24}{\log k}}
$$

$A$ étant une constante. Il suffit de prendre la constante $k$ suftisamment grande pour que $\frac{\log 24}{\log k}$ soit inférieur à l'ordre $\varrho$ de la fonction $f(z)$, pour entraîner la contradiction.

Nous déterminerons la couronne circulaire $(C)$ par les inégalités:

$$
r_{n}<|z|<r_{n} \cdot e^{\frac{6}{e}}
$$

et d'après ce qui précède, nous pourrons choisir une suite de valeurs de $r_{n}$ tendant vers l'infini, pour lesquelles:

$$
\lim _{n=\infty} \frac{\log T\left(r_{n}\right)}{\log r_{n}}=\varrho .
$$

L'application du théorème IV fournit alors le théorème suivant:

VI. Soit $f(z)$ une fonction méromorphe d'ordre fini $\varrho$, ̀̀ fonction caractéristique $T(r)$. Étant donné un nombre $q$, il existe une suite $r_{1}, r_{2}, \ldots r_{n}, \ldots$ de valeurs de $r$ tendant vers linfini, et pour lesquelles:

$$
\lim _{n \rightarrow \infty} \frac{\log T\left(r_{n}\right)}{\log r_{n}}=\varrho
$$

telles que sur chaque cercle de centre o et de rayon $r_{n}$, se trouve un point, centre d'un cercle de remplissage $C_{n}(q)$, de rayon $\frac{r_{n}}{q}$, à l'intérieur duquel le nombre des zéros de $f(z)-a$ est supérieur à

27 - 2822. Acta mathematica. 52. Imprimé le 17 septembre 1928. 


$$
n_{1}=\frac{A}{q^{2}} T\left(r_{n}\right)
$$

sauf peut-être pour des valeurs de a dont les représentations sphériques sont incluses dans deux petits cercles de rayons $e^{-n_{1}}$.

$A$ est une constante dépendant uniquement de la fonction $f(z)$.

17. La limite inférieure $n_{1}$ du nombre des zéros de $f(z)-a$ contenus dans le cercle de remplissage $C_{n}(q)$ est d'une précision à peu près définitive, comme le montre l'exemple des fonctions elliptiques: pour de telles fonctions, en effet, le nombre des zéros de $f(z)-a$ contenus dans tout cercle dont le centre est situé à la distance $r$ de l'origine, et dont le rayon a pour valeur $\frac{r}{q}$, est inférieur à:

$$
\frac{B}{q^{2}} \cdot T(r)
$$

$B$ désignant une constante dépendant uniquement de la fonction elliptique envisagée.

On peut dire qu'étant donné un cercle de remplissage, la limite inférieure du nombre des zéros de $f(z)-a$ situés dans ce cercle, déterminée par le théorème VI, est vraie à un facteur constant près. Ces résultats sont en progrès notable sur les résultats analogues, dus à M. Valiron ${ }^{1}$, et dans lesquels la limite inférieure du nombre des zéros de $f(z)-a$ situés dans le cercle de remplissage est de la forme:

$$
\frac{A}{q^{2}} \frac{T\left(r_{n}\right)}{\log \left(r_{n}\right)}
$$

I8. L'énoncé du théorème VI se précise dans le cas de fonctions méromorphes à croissance très régulière, c'est-à-dire de fonctions pour lesquelles on a l'inégalité:

$$
h_{1} r^{\circ}<T(r)<h_{2} r^{\circ}
$$

$h_{1}$ et $h_{2}$ sont des constantes positives. Pour de telles fonctions, le rapport de deux $r_{n}$ consécutifs est borné, et dépend du rapport $h_{2}: h_{1}$; comme couronne circulaire $(C)$, on peut en effet prendre toute couronne définie par les inégalites:

${ }^{1}$ G. Valiron. Recherches sur le théorème de $M$. Borel dans la théorie des fonctions méromorphes (Acta math., t. 52, 1928, p. 67). 


$$
r<|z|<r\left(\frac{24 h_{2}}{h_{1}}\right)^{\frac{2}{\rho}}
$$

19. Nous avons obtenu le théorème VI en donnant, dans l'application du théorème IV, une valeur invariable à la constante $q$. On peut aussi appliquer ce théorème en considérant $q$ comme fonction du rang $n$ du cercle de remplissage $C_{n} ; q$ pourra ainsi tendre vers l'infini avec $n$, pas trop rapidement, cependant, pour que la première condition énoncée dans le théorème IV ne cesse d'être vérifiée. Ce choix de $q$ permet d'obtenir des cercles de remplissage vus de l'origine sous des angles tendant vers zéro lorsque les distances de leurs centres à l'origine tendent vers l'infini.

$1 l$ est.possible, par exemple, de fixer pour valeur de $q(n)$ une fonction de $n$ tendant vers l'infini avec $n$, d'une facon suffisamment lente pour que le nombre des zéros de $f(z)-a$ situés dans le cercle de remplissage $C_{n}$ soit supérieur à:

$$
r_{n}^{\rho-\varepsilon(n)}
$$

$\varepsilon(n)$ étant une fonction tendant vers zéro avec $\frac{\mathrm{I}}{n}$, et ceci, pour toute valeur de a, sauf peut-être pour des valeurs dont les représentations sphériques sont incluses dans deux petits cercles dont les rayons tendent vers zéro avec $\frac{\mathrm{I}}{n}$.

On peut aisément préciser la valeur de $\varepsilon(n)$ en fonction de $T\left(r_{n}\right)$ et de $q(n)$. Sous sa forme imprécisée, l'énoncé précédent est identique à celui qui a été obtenu. par M. Valiron, dans son mémoire déjà cité, et qui a conduit immédiatement cet auteur à la démonstration de l'existence de courbes de Borel. En effet, dans une suite de cercles de remplissage s'éloignant. indéfiniment, et satisfaisant à l'énoncé précédent, l'exposant de convergence de la suite des zéros de $f(z)-a$ est égal à $\varrho$, sauf pour deux valeurs au plus de a.

La courbe, se déduisant par rotation d'une courbe fixe, et dont la position est définie par une des valeurs limites des arguments curvilignes des centres d'une suite de ces cercles de remplissage, est ce que $\mathbf{M}$. Valiron a appelé une courbe de Borel. Une rotation arbitrairement petite, dans un sens et dans l'autre, lui fait balayer un domaine contenant une infinité de cercles de remplissage.

20. Pour déceler l'existence de courbes de Borel, il est nécessaire de prendre des cercles de remplissage vus de l'origine sous des angles tendant vers zéro d'une façon assez lente. Dans un autre ordre d'idées, on peut se proposer de rechercher 
la limite inférieure du rayon du cercle de remplissage. L'application du théorème IV fournit immédiatement l'énoncé suivant:

VII. Soit $f(z)$ une fonction méromorphe d'ordre @, à fonction caractéristique $T(r)$. Il existe une suite de valeurs $r_{1}, r_{2}, \ldots r_{n}, \ldots$ tendant vers l'infini, et pour lesquelles:

$$
\lim \frac{\log T\left(r_{n}\right)}{\log r_{n}}=\varrho
$$

telles que sur chaque cercle $|z|=r_{n}$ se trouve un point centre d'un cercle de remplissage $O_{n}$ de rayon:

$$
\frac{k r_{n}}{A_{n} \frac{\sqrt{T\left(r_{n}\right)}}{T}}
$$

à l'intérieur duquel la fonction $f(z)$ prend toute valeur, sauf peut-être des valeurs dont les représentations sphériques sont incluses dans dewx petits cercles de rayon $e^{-A_{n}}$.

$k$ est une constante dépendant uniquement de la fonction $f(z)$ envisagée, et $A_{n}$ est une quantité positive arbitraire, à laquelle on donnera une valeur tendant vers l'infini avec $n$, de façon que le théorème de Picard soit vérifié dans une suite de cercles de remplissage s'éloignant indéfiniment.

La valeur du rayon du cercle de remplissage, fournie par ce théorème VII, précise une valeur obtenue précédemment par M. Valiron. ${ }^{1}$ L'exemple des fonctions elliptiques montre encore que cette valeur est exacte à un facteur constant près, ce qui est très précis.

2I. Comme l'a montré M. Valiron dans le mémoire qui vient d'être cité, le cas des fonctions entières se distingue très nettement de celui des fonctions méromorphes, et les valeurs minima des rayons des cercles de remplissage sont très différentes. D'autre part, dans l'énoncé du théorème VI, on signale la possibilité de valeurs exceptionnelles, tandis que dans l'exemple des fonctions elliptiques, ces valeurs exceptionnelles n'existent pas; nous verrons plus tard que la valeur minima du rayon du cercle de remplissage qui est valable pour les fonctions entières, est également valable pour une fonction méromorphe d'ordre fini, possédant, dans certains domaines, une valeur exceptionnelle au sens large du mot.

22. Dans l'étude des cercles de remplissage des fonctions méromorphes d'ordre infini, on utilise également les inégalités (3) et (4). La méthode de M.

1 G. Valiron. Compléments au théorème de Picard-Julia (Bull. des Sc. Math., t. 5 I, 1927, p. 167). 
Borel permet d'éliminer, dans $S(r)$, les termes en $\log T(R)$ et $\log _{\frac{+}{R--}} \frac{1}{r}$. Nous allons voir qu'il existe un système de nombres $(r, R)$ pour lesquels:

$$
24 \log T(R)+\mathrm{I} 2 \stackrel{+}{\log } \frac{\mathrm{I}}{R-r}<\frac{\mathrm{I}}{2} T(r) .
$$

Supposons en effet qu'il n'en soit pas ainsi, à partir d'une certaine valeur $r_{1}$ de $r$ et déterminons la suite $r_{1}, r_{2}, \ldots r_{n}, \ldots$ par les égalités suivantes:

$$
\begin{gathered}
r_{2}=r_{1}+\frac{\mathrm{I}}{T\left(r_{2}\right)} \\
\cdot . \cdot \cdot \cdot \\
r_{n}=r_{n-1}+\frac{\mathrm{I}}{T\left(r_{n}\right)} .
\end{gathered}
$$

D'après l'hypothèse, on a:

$$
\log T\left(r_{n}\right)>\frac{1}{72} T\left(r_{n-1}\right)
$$

Partons de $n=2$ et étudiọns la suite des valeurs $T\left(r_{n}\right)$ : d'après l'inégalité précédente, si $T\left(r_{1}\right)$ dépasse une constante numérique, on a:

et par suite:

$$
T\left(r_{2}\right)>2 T\left(r_{1}\right)
$$

$$
T\left(r_{3}\right)>2 T\left(r_{2}\right)
$$

et ainsi de suite, de sorte que:

$$
T\left(r_{n}\right)>2^{n} T\left(r_{1}\right)
$$

et, d'après la loi de récurrence choisie pour déterminer $r_{n}$, cette dernière quantité est inférieure à:

$$
r_{1}+\frac{2}{T\left(r_{1}\right)}
$$

quel que soit $n$, alors que $T\left(r_{n}\right)$ tend vers l'infini avec $n$.

Un tel résultat est impossible, et l'on peut affirmer qu'entre $r$ et $r+\frac{2}{T(r)}$, il existe un système de nombres $\left(r^{\prime}, R^{\prime}\right)$ tel que:

$$
24 \log T\left(R^{\prime}\right)+\mathrm{I} 2 \log \frac{\mathrm{I}}{R^{\prime}-r^{\prime}}<\frac{\mathrm{I}}{2} T\left(r^{\prime}\right) .
$$


On pourra par suite écrire la deuxième inégalité de M. R. Nevanlinna sous la forme suivante:

$$
\begin{aligned}
\frac{\mathrm{I}}{2} T\left[r-\frac{2}{T(r-\mathrm{I})}\right]<N(r, a)+N(r, b) & +N(r, c)+8 \log ^{+} \\
& +c_{2}(f)+8 \log ^{+} \frac{\mathrm{I}}{|a-b||b-c||c-a|} .
\end{aligned}
$$

L'étude se poursuit de la même façon que pour les fonctions méromorphes d'ordre fini ou d'ordre nul, et on aboutit au théorème suivant, analogue au théorème $1 \mathrm{~V}$ :

VIII. - Soit $f(z)$ une fonction méromorphe d'ordre infini, à fonction caractéristique $T(r)$. Dans la couronne circulaire $(C)$ :

$$
r<|z|<R
$$

il existe un cercle de remplissage $\left(C_{i}\right)$ de rayon $\frac{\mathrm{IOO} r_{i}}{q}, r_{i}$ désignant le module de l'affixe du centre de $\left(C_{i}\right)$, dans lequel la fonction $f(z)$ prend plus de:

$$
n_{1}=\frac{\mathrm{I}}{\mathrm{I} 8 \mathrm{OO} q^{2}} \frac{T\left[R-\frac{2}{T(R-\mathrm{I})}\right]}{\log \frac{R}{r}}
$$

fois toute valeur, sauf peut-être des valeurs dont les représentations sphériques sont incluses dans deux petits cercles de rayon $e^{-n_{x}}$.

Les conditions d'application sont les suivantes:

I. $n_{1}$ et $q$ doivent dépasser des constantes numériques.

2. $T\left[R-\frac{2}{T(R-\mathrm{I})}\right]$ doit être supérieur aux trois quantités suivantes:

$$
c_{3}(f), \quad 24 T(r), \quad 24 \frac{T(k r)}{\log k} \log \frac{R}{r}
$$

$c_{3}(f)$ est une constante dépendant uniquement de la fonction $f(z) ; k$ est un nombre quelconque compris entre I et $\frac{R}{r}$.

Il est clair qu'il existe en particulier des couronnes circulaires $(C)$ d'épaisseur relative finie, abitrairement fixée à l'avance. On en déduit l'existence de courbes analogues aux courbes de Borel relatives aux fonctions méromorphes d'ordre fini. 
Les cercles de remplissage des fonctions méromorphes ou entières.

23. Nous allons faire maintenant l'étude, annoncée au $\mathrm{n}^{\circ} 2 \mathrm{I}$, d'une fonction méromorphe admettant, dans certains domaines, une valeur exceptionnelle au sens large. Nous ramènerọns, d'une façon générale, à l'étude d'une fonction méromorphe $g(x)$ dans le cercle $|x|=\mathrm{I}$. On suppose que, dans le cercle $|x|=\frac{\mathrm{I}}{2}$, la fonction prend plus de $n_{1}$ fois toute valeur, sauf peut-être des valeurs dont les représentations sphériques sont incluses dans deux cercles de rayon $e^{-n_{1}}$, et que dans le cercle $|x|=\mathrm{I}$, la fonction ne prend pas plus de $\alpha n_{1}$ fois une valeur que nous pourrons supposer infinie; $\alpha$ est une constante numérique que nous fixerons dans le cours du raisonnement.

Soient $a_{1}, a_{2}, \ldots a_{n}, \ldots$ les pôles de $g(x)$ situés dans le cercle $|x|=\mathrm{I}$. La fonction holomorphe:

$$
\varphi(x, a)=[g(x)-a] \Pi\left(x-a_{n}\right)
$$

possède, dans le cercle $|x|=\frac{I}{2}$, un nombre de zéros supérieur à $n_{1}$, et ceci, pour un très grand choix de valeurs de $a$ (nous prendrons ces valeurs de modules inférieurs à un). Pour cette fonction $\varphi(x, a)$, on a:

$$
\begin{gathered}
N\left(\frac{5}{8}, 0\right)>\int_{\frac{1}{2}}^{\frac{5}{8}} n\left(\frac{\mathrm{I}}{2}, 0\right) \frac{d t}{t}>\frac{2}{9} n_{1} \\
T\left(\frac{5}{8}\right)>\frac{2}{9} n_{1}+h\left(\frac{5}{8}\right)
\end{gathered}
$$

avec:

$$
\left|h\left(\frac{5}{8}\right)\right|<\log 2+\stackrel{+}{\log } \frac{\mathrm{I}}{|\varphi(o, a)|} .
$$

Deux cas sont à considérer, suivant les valeurs de $\varphi(x)$ aux environs de l'origine.

Premier cas. - Il existe un point $x_{0}$, de module inférieur à $\frac{\mathrm{I}}{8}$, en lequel:

$$
\log ^{+} \frac{\mathrm{I}}{\left|\varphi\left(x_{0}, a\right)\right|}<\frac{n_{1}}{\text { IO }}
$$

Un déplacement d'origine conduit à l'inégalité:

$$
T\left(\left|x-x_{0}\right|=\frac{25}{32}\right)>\frac{n_{1}}{9}
$$


dès que $n_{1}$ dépasse une certaine constante numérique. On en déduit aussitôt, d'après une inégalité due à M. R. Nevanlinna, la limitation suivante:

$$
\log M\left(\frac{29}{32}\right)>\frac{n_{1}}{9}
$$

$M\left(\frac{29}{32}\right)$ désigne le maximum de $|\varphi(x, a)|$ sur le cercle $|x|=\frac{29}{32}$.

Deuxième cas. - En tout point $x$ de module inférieur à $\frac{\mathrm{I}}{8}$, on $\mathbf{a}$ :

$$
\stackrel{+}{\log } \frac{\mathrm{I}}{|\varphi(x, a)|}>\frac{n_{1}}{\mathrm{IO}}
$$

Nous allons en déduire que, en choisissant convenablement la constante $\alpha$ qui n'a pas encore été précisée, la fonction $g(x)-a$ est petite sur un cercle intérieur au cercle $|x|=\frac{\mathrm{I}}{8}$. D'après le théorème de Boutroux, on $\mathrm{a}$ :

$$
\Pi\left|x-a_{n}\right|>k^{\alpha n_{1}}
$$

à l'extérieur de couronnes circulaires de centre o et d'épaisseur totale $2 e k$. Si nous prenons $2 e k$ inférieur à $\frac{1}{8}$, il existe une circonférence $(\gamma)$, intérieure à la circonférence $|x|=\frac{I}{8}$, sur laquelle l'inégalité précédente est vérifiée. Un calcul simple établit que si l'on prend $\alpha=\frac{1}{80}$, la fonction $g(x)$ vérifie, sur la circonférence $(\gamma)$, l'inégalité:

$$
|g(x)-a|<e^{-\frac{n_{1}}{20}}
$$

La circonférence $(\gamma)$ est indépendante de la valeur $a$; par conséquent, si le deuxième cas se présente pour une valeur $a$, il suffit de considérer la fonction $\varphi(x, b)$ pour retomber sur le premier cas, $b$ n'étant pas trop proche de $a$; un tel choix est d'ailleurs possible d'après ce que nous avons vu plus haut.

En résumé, il est toujours possible de choisir une valeur $a$ de module inférieur à un, de telle façon que la valeur de $|\varphi(x, a)|$ en un point $P$ du cercle $|x|=\frac{29}{32}$ soit supérieure à $e^{\frac{n_{1}}{9}}$. La fonction $\varphi(x, a)$ étant holomorphe, on en déduit que cette propriété est valable, non seulement au point $P$, mais encore sur une courbe $(\Gamma)$ traversant la couronne circulaire: 


$$
\frac{29}{32}<|x|<\mathrm{I}
$$

Une inégalité analogue est valable pour la fonction $g(x)$. On a en effet:

$$
\Pi\left|x-a_{n}\right|<e^{\frac{n_{1}}{100}}
$$

et, par suite, sur la courbe $(r)$ :

$$
|g(x)|>e^{\frac{n_{1}}{10}}
$$

24. Nous allons déduire de cette propriété l'existence d'un cercle de remplissage: opérons la représentation conforme du cercle $|x|=\mathrm{I}$ sur le cercle $\left|x^{\prime}\right|=\mathrm{r}$, le point $P$ correspondant au centre $P^{\prime}$ du nouveau cercle. Soit $x_{1}^{\prime}$ le point le plus proche de $P^{\prime}$, en lequel le module de $g\left(x^{\prime}\right)$ est égal à un. A l'intérieur du cercle $\left|x^{\prime}\right|=\left|x_{1}^{\prime}\right|$, la fonction $g\left(x^{\prime}\right)$ est supérieure en module à un, et, sur une courbe $\left(\Gamma^{\prime}\right)$ traversant ce cercle, on a:

$$
\left|g\left(x^{\prime}\right)\right|>e^{\frac{n_{1}}{10}}
$$

Il résulte alors d'une inégalité de M. Carleman qu'en tout point $x^{\prime}$ de module inférieur à $\left|x_{1}^{\prime}\right|$, l'inégalité:

$$
\log \left|g\left(x^{\prime}\right)\right|>\frac{\lambda n_{1}}{\mathrm{IO}}\left(\mathrm{I}-\left|\frac{x^{\prime}}{x_{1}^{\prime}}\right|\right)
$$

est vérifiée, $\lambda$ étant une constante numérique.

Construisons le cercle $(\gamma)$ passant par $x_{1}^{\prime}$, et de rayon $\frac{\operatorname{IO} A\left|x_{1}{ }^{\prime}\right|}{n_{1} \lambda}$, et le cercle $\gamma^{\prime}$ concentrique et de rayon double. Sur une courbe traversant la couronne circulaire limitée par ces deux cercles, le module de $g\left(x^{\prime}\right)$ est supérieur à $e^{A}$; d'après un théorème démontré dans ma thèse, la fonction $g\left(x^{\prime}\right)$ prend, dans le cercle $(\gamma)$, toute valeur, sauf peut-être des valeurs dont les représentations sphériques sont incluses dans deux petits cercles de rayon $e^{-\mu \Lambda}, \mu$ étant une constante numérique.

25. Retournons à la fonction méromorphe $g(x)$ dans le cercle $|x|=\mathbf{I}$, en supposant à cette fonction une valeur exceptionnelle quelconque, au lieu de l'infini. La transcription des résultats précédents fournit la proposition suivante:

IX. Soit $g(x)$ une fonction méromorphe dans le cercle $|x|=R$, et ne prenant 28-2822. Actu mathematica. 52. Imprimé le 18 septembre 1928. 
pas, dans ce cercle, plus de $\frac{n_{1}}{80}$ fois la valeur a; on suppose que, dans le cercle $|x|=\frac{R}{2}$, la fonction $g(x)$ prend plus de $n_{1}$ fois toute valeur, sauf peut-être des valeurs dont les représentations sphériques sont incluses dans deux petits cercles de rayon $e^{-n_{1}}$. La fonction $g(x)$ vérifie alors les propriétés suivantes:

$I^{\circ}$. Sur une courbe $(\Gamma)$ traversant la couronne.

$$
\frac{29}{32} R<|z|<R
$$

les distances sphériques qui séparent de a les valeurs que prend la fonction $g(x)$ sont inférieures à $e^{-\frac{n_{1}}{10}}$.

$2^{\circ}$. Il existe, à l'intérieur du cercle $|x|=R$, un cercle de remplissage de rayon $\frac{A R}{n_{1}}$ à l'intérieur duquel la fonction $g(x)$ prend toute valeur, sauf peut-être des valeurs dont les représentations sphériques sont incluses dans deux petits cercles de rayon $e^{-\lambda \Delta} \lambda$ étant une constante numérique.

Les quantités $n_{1}$ et $A$ doivent dépasser des constantes numériques.

26. Le théorème IX peut s'appliquer à des fonctions méromorphes dans tout le plan; son application aux fonctions méromorphes d'ordre fini conduit de suite à la proposition suivante, qui complète le théorème VI:

X. Si, dans le cercle concentrique $\grave{a} C_{n}(q)$ et de rayon double, la fonction $f(z)$ ne prend pas plus de $\frac{n_{1}}{80}$ fois une valeur a, il existe un cercle de remplissage $\left(\gamma_{n}\right)$ intérieur à $\left(C_{n}\right)$, de rayon $\frac{B r_{n}}{q n_{1}}$, dans lequel la fonction $f(z)$ prend toute valeur, sauf peut-être des valeur's dont les représentations sphériques sont incluses dans deux petits cercles de rayon $e^{-B \lambda}, \lambda$ étant une constante numérique.

Il en résulte que si $q$ a une valeur inférieure à une constante indépendante de $n$, pour une suite de cercles $C_{n}(q)$, il existe des cercles de remplissage $\left(\gamma_{n}\right)$ de rayons $B \mu \frac{u_{n}}{T\left(u_{n}\right)}, u_{n}$ désignant le module de l'affixe du centre du cercle $\left(\gamma_{n}\right)$ et $\mu$ une constante numérique.

Cette valeur $d u$ rayon du cercle de remplissage est inférieure à la valeur minima donnée par le théorème VII. L'exemple de la fonction entière $e^{z}$ montre, qu'à part la valeur du facteur numérique $\mu$, elle est exacte, et ne peut être rendue plus petite. 
27. Le théorème $\mathrm{X}$ étend la valeur minima du rayon du cercle de remplissage d'une fonction entière ${ }^{1}$, au cas d'une fonction méromorphe possédant, dans certaines régions, une valeur exceptionnelle au sens large du mot. Par exemple, on précisera l'énoncé en considérant une fonction méromorphe d'ordre fini, possédant une valeur exceptionnelle $a$ dans l'une des couronnes circulaires (C) du théorème III, le nombre des zéros de $f(z)-a$ devant être inférieur à

$$
k \frac{T(R)}{\log \frac{R}{r}}
$$

$k$ est une constante numérique; $\log \frac{R}{r}$ est aussi une constante, mais qui dépend de l'ordre $\varrho$ de la fonction méromorphe $f(z)$.

Le théorème $X$ s'étend aussi aux fonctions d'ordre infini, avec, comme valeur du rayon du cercle de remplissage:

$$
B \mu \frac{u_{n}}{T\left[u_{n}-\frac{2}{T\left(u_{n}-1\right)}\right]}
$$

28. Les applications précédentes du théorème IX aux fonctions méromorphes d'ordre fini ou infini ont lieu, soit dans des cercles de remplissage, soit dans des couronnes circulaires $(C)$ d'épaisseur relative finie, dans lesquelles d'ail leurs se trouvent des cercles de remplissage.

On pourra aussi appliquer le théorème IX à l'étude des fonctions méromorphes d'ordre nul dans des cercles de remplissage (lorsque la fonction possède de tels cercles et, dans ces cercles, une valeur exceptionnelle au sens large).

Mais l'application du théorème IX aux couronnes circulaires $(C)$ est moins interessante, car l'épaisseur relative de ces couronnes tend vers l'infini lorsqu'elles s'éloignent indéfiniment. Donnons seulement quelques indications sur la méthode à suivre dans cette étude:

On effectue d'abord l'étude d'une fonction méromorphe $g(x)$ dans le cercle $|x|=\mathrm{I}$. On suppose que cette fonction, dans le cercle $|x|=\varrho$ de rayon $\varrho$ inférieur à un, prend $n_{1}$ fois toute valeur, sauf peut-être des valeurs dont les représentations sphériques sont incluses dans deux petits cercles de rayon $e^{-n_{1}}$,

${ }^{1}$ G. Valiron. Compléments au théorème de Picard-Julia (dejà eité). 
tandis que, dans le cercle $|x|=\mathrm{I}$, le nombre des zéros de $g(x)-a$ est inférieur à $\alpha n_{1}$.

L'étude s'effectue d'une manière analogue à celle des numéros 23 et 24 ; mais ici $\alpha$, au lieu d'être une constante numérique, est une fonction de $\varrho$ tendant vers zéro avec $I-\varrho$.

Ensuite, étant donnée une fonction $f(z)$, méromorphe dans la couronne circulaire :

$$
\frac{r}{2}<|z|<2 R
$$

on effectue la représentation conforme d'un secteur de cette couronne circulaire, d'ouverture $2 \pi$ par exemple, sur le cercle $|x|=\mathrm{I}$, de façon que le domaine correspondant au cercle $|x|=\varrho$ contienne à son intérieur la moitié de la couronne circulaire $(C)$ déterminée par les inégalités:

$$
r<|z|<R
$$

ce qui fixe une dépendance entre $\varrho$ et $\frac{R}{r}$.

Le nombre $n_{1}$ est supérieur à la moitié du nombre des zéros de $f^{\prime}(z)-b$ (sauf pour des valeurs exceptionnelles possibles de $b$ ) situés dans la couronne circulaire $(C)$, et par suite à:

$$
\frac{A T(R)}{\log \frac{R}{r}}
$$

$A$ étant une constante numérique.

On obtient ainsi un cercle de remplissage, à condition de supposer que dans la couronne circulaire:

$$
\frac{r}{2}<|z|<2 R
$$

le nombre des zéros de $f(z)-a$ est inférieur, pour une valeur de $a$, à une quantité de la forme: $\alpha\left(\frac{R}{r}\right) n_{1} ; \alpha\left(\frac{R}{r}\right)$ est une fonction de $\frac{R}{r}$ seulement, tendant vers zéro lorsque $\frac{R}{r}$ tend vers l'infini. On constate que les cercles de remplissage ainsi trouvés sont vus de l'origine sous des angles qui tendent vers zéro lorsque la couronne circulaire $(C)$ s'éloigne indéfiniment. 


\section{DEUXIEME PARTIE. \\ Fonctions entières d'ordre fini.}

CHAPITRE III.

\section{Étude angulaire des domaines où une fonction entière est très grande.}

29. Soit $f(z)$ une fonction entière, dont le module maximum sur le cercle $|z|=r$ est désigné par $e^{V(r)}$, et soit $M$ un point, de module $r$, en lequel la fonction $f(z)$ vérifie l'inégalité:

$$
\log |f(z)| \geq \mid z p^{\prime} .
$$

Le domaine des points où la fonction $f(z)$ vérifie l'inégalité:

$$
\log |f(z)| \geq \varepsilon|z|^{\prime}
$$

où $\varepsilon$ est une quantité positive inférieure à un, comprend un domaine $A$, d'un seul tenant, qui contient à son intérieur le point $M$. Nous nous proposons de montrer que l'ouverture angulaire de ce domaine $A$, limité à l'intérieur de la couronnè circulaire:

$$
r_{1} \leq|z| \leq r_{2}
$$

est au moins égale à:

$$
\frac{\pi}{\varrho^{\prime}\left(I+\varepsilon^{\prime}\right)}
$$

certaines inégalités étant vérifiées entre les quantités $r, r_{1}, r_{2}, \varepsilon, \varepsilon^{\prime}$.

3o. Supposons done l'ouverture angulaire du domaine $A$ inférieure à $\frac{\pi}{\varrho^{\prime}\left(\mathrm{I}+\varepsilon^{\prime}\right)}$, et désignons par $(A)$ le secteur de la couronne:

$$
r_{1} \leq|z| \leq r_{2}
$$

qui a pour ouverture $\frac{\pi}{\varrho^{\prime}\left(1+\varepsilon^{\prime}\right)}$, et qui contient le domaine $A$. Une rotation nous permettra de supposer ce secteur disposé symétriquement par rapport à l'axe réel.

Sur la partie du contour du domaine $A$ située à l'intérieur du secteur $(A)$, la fonction $f(z)$ vérifie l'égalité:

$$
\log |f(z)|=\varepsilon|z|^{\prime}
$$


et, par suite, l'inégalité:

$$
|f(z)|<\left|e^{\frac{1}{3} z \dot{Q}^{\prime}}\right|
$$

si l'on a dans le secteur $(A)$ :

$$
\varepsilon|z|^{\rho^{\prime}}<\frac{1}{3} \mathfrak{R}\left(z^{\varrho^{\prime}}\right)
$$

Or:

$$
\Re\left(z^{0^{\prime}}\right) \geq|z|^{\rho^{\prime}} \cos \frac{\pi}{2\left(\mathrm{I}+\varepsilon^{\prime}\right)}
$$

Pour que l'inégalité (IO) soit vérifiée, il suffit donc de prendre $\varepsilon^{\prime}=3 \varepsilon$ et de supposer $\varepsilon$ inférieur à $\frac{\mathrm{I}}{6}$.

Ceci posé, considérons la fonction:

$$
\varphi(z)=f(z) e^{-V^{\prime}\left(r_{1}\right)-\sigma q^{Q^{\prime}}\left(1+\frac{3}{2} \varepsilon\right)}
$$

$\sigma$ étant une constante positive que nous déterminerons dans la suite.

Dans le secteur $(A)$, la partie réelle de $z^{\rho^{\prime}\left(1+\frac{3}{2} \varepsilon\right)}$ est positive et au moins égale à:

$$
\varepsilon|z| p^{\prime}\left(1+\frac{3}{2} \varepsilon\right) \text {. }
$$

Le module de la fonction $\varphi(z)$ est évidemment inférieur à un en tout point du domaine $\Delta$ situé sur la circonférence $|z|=r_{1}$. Il en est de même en tout point du domaine $\Delta$ situé dans le secteur $(A)$, et sur la circonférence $|z|=r_{2}$, si l'on a soin de fixer pour valeur de $\sigma$ :

$$
\sigma=-\frac{V\left(r_{2}\right)}{\varepsilon r_{2}^{Q^{\prime}}\left(1+\frac{3}{2} \varepsilon\right)} .
$$

Des inégalités précédentes, il résulte alors que sur la frontière de la partie du domaine $\Delta$ située à l'intérieur et sur la frontière du secteur $(A)$, et par suite en tout point intérieur à cette partie, on a l'inégalité:

$$
|\varphi(z)|<\left|e^{\frac{1}{3} z e^{\prime}}\right|
$$

et par suite:

$$
|f(z)|<\left|e^{V\left(r_{1}\right)+\frac{1}{3} z Q^{\prime}+\sigma z Q^{\prime}\left(1+\frac{8}{2} \varepsilon\right)}\right| .
$$

Une telle inégalité est contrađictoire avec l'hypothèse faite sur la valeur de $|f(z)|$ au point $M$ de module $r$, si l'on a les deux inégalités: 


$$
V\left(r_{1}\right)<\frac{\mathrm{I}}{3} r^{e^{\prime}}
$$

et:

$$
V\left(r_{2}\right)<\frac{\varepsilon}{3} r_{2}^{e^{\prime}}\left(\frac{r_{2}}{r}\right)^{\frac{8}{2} \varepsilon \rho^{\prime}}
$$

d'où le théorème suivant:

XI. - Soit $f(z)$ une fonction entière, supérieure en module à $e^{r e^{\prime}}$ en un point $M$ de module $r$. Les domaines des points où la fonction $f(z)$ vérifie l'inégalité:

$$
\log |f(z)|>\varepsilon|z|^{\prime}
$$

comprennent un domaine ouvert $\boldsymbol{A}$, d'un seul tenant, contenant à son intérieur le point $M$, et tel que les arguments extrêmes (déterminés par continuité) des points du domaine $\triangle$ situés dans la couronne:

$$
r_{1} \leq|z| \leq r_{z}
$$

diffèrent de plus de:

$$
\frac{\pi}{\varrho^{\prime}(\mathrm{I}+3 \varepsilon)}
$$

Les conditions d'application sont les suivantes:

$$
\left\{\begin{array}{l}
V\left(r_{1}\right) \leq \frac{\mathbf{I}}{3} \cdot \cdot^{\prime} \\
V\left(r_{2}\right) \leq \frac{\varepsilon}{3} r_{2}^{\sigma^{\prime}}\left(\frac{r_{2}}{r}\right)^{\frac{3}{2} \varepsilon \rho^{\prime}}
\end{array}\right.
$$

$V(r)$ désigne le maximum de log $|f(z)|$ sur le cercle $|z|=r$.

3I. Cette propriété d'extension angulaire du domaine $\Delta$ est susceptible d'un énoncé un peu plus général: nous avons supposé en effet que la fonction $f(z)$ est une fonction entière. Nous aurions pu prendre une fonction holomorphe dans un angle. Dans ce cas, l'extension angulaire du domaine $\Delta$ est la même que plus haut, à moins que la partie considérée du domaine $\Delta$ ne soit coupée par l'un des côtés de l'angle dans lequel la fonction est holomorphe.

32. D'après les inégalités (I I), on pourra toujours choisir une valeur de $r_{1}$ assez petite pour que la première inégalité soit vérifiée. Mais la deuxième inégalité limite le choix de $\varrho^{\prime}$, suivant les propriétés de la fonction $V(r)$. 
Ainsi, lorsque la fonction $f(z)$ est une fonction entière d'ordre infini, on peut donner à $\varrho^{\prime}$ des valeurs dépendant de $r$, et tendant vers l'infini avec $r$, à condition que la fonction $\frac{\log V(r)}{\log r}$ soit décroissante dans certains intervalles. Nous utiliserons plus loin cette remarque.

33. Dans le cas d'une fonction entière d'ordre fini $\varrho$, on pourra par exemple choisir:

$$
\begin{aligned}
\varrho^{\prime} & =\varrho(\mathrm{I}-\eta) \\
\varepsilon & =\frac{3 \varepsilon \eta}{\varepsilon^{\prime}} \\
r_{2} & =r^{1+\varepsilon^{\prime}} \\
r_{1} & =\gamma^{1-3 \eta}
\end{aligned}
$$

$\eta$ étant une constante positive. On pourra même assigner à $\eta$ une valeur fonction de $r$ tendant vers zéro avec $\frac{r}{r}$, de manière que les inégalités:

$$
r^{(1-\eta)}<\log |f(z)|<r^{(1+\eta)}
$$

soient vérifiées, la deuxième à partir d'une certaine valeur de $r$, la première (limitation inférieure de $\log |f(z)|)$ pour une suite de valeurs de $r=|z|$ tendant vers l'infini.

Sous cette forme particulière, le théorème XI précise certaines propriétés de croissance d'une fonction entière d'ordre fini $\varrho$ supérieur à $\frac{1}{2}$, propriétés que j'ai établies dans ma thèse ${ }^{1}$. Par exemple, on en déduit l'existence d'un angle d'ouverture $\frac{\pi}{\varrho}$, tel que sur toute demidroite intérieure à cet angle, la fonction $f(z)$ est d'ordre $\varrho$.

M. Valiron a obtenu récemment d'autres propriétés présentant une certaine analogie avec les précédents: il fixe un minimum de $|f(z)|$ sur certains arcs de cercles de centre $o$, et dont l'ouverture dépend du plus grand entier contenu dans $\varrho .^{2}$

${ }^{1} \mathrm{H}$. Milloux. Le théorème de M. Picard... (déjà cité). Ces propriétés sont déduites du principe de Phragmén-Lindelöf. En somme, la démonstration du théorème XI est inspirée d'une précision de ce célèbre principe. J'ai étendu, dans ma thèse, ces propriétés à des angles curvilignes. On pourrait donner une pareille extension du théorème XI.

${ }^{2}$ G. Valiron. Sur quelques propriétés des fonctions entières (Compte-Rendus, t. I85, I927, p. I439). 
34. Etudions plus particulièrement l'application du théorème XI aux fonctions entières à croissance très régulière, pour lesquelles:

$$
(h r) e \leq V(r) \leq(k r) e
$$

$h$ et $k$ étant deux constantes positives.

En effectuant la transformation $Z=k z$, la fonction $f(z)$ devient une fonction très régulière de $Z$, d'ordre $\varrho$, et sur tout cercle $|Z|=R$, il existe un point où l'on $a$ :

$$
\log |f(Z)|=|Z| e
$$

On peut donc appliquer le théorème XI en faisant $\varrho^{\prime}=\varrho$; en retournant à la fonction $f(z)$, on obtient la proposition suivante:

XII. Soit $f(z)$ une fonction entière, telle que la maximum $V(r)$ de $\log |f(z)|$ sur le cercle $|z|=r$ vérifie, quel que soit $r$ l'inégalité:

$$
(h r) e \leq V(r) \leq(k v) e
$$

$h$ et $k$ étant dewx constantes positives.

Les domaines des points où la fonction $f(z)$ vérifie l'inégalité:

$$
\log |f(z)|>\varepsilon(h r) e
$$

comprennent, dans toute couronne circulaire $\Gamma$ défnie par les inégalités:

$$
r \frac{h}{l}\left(\frac{\mathrm{I}}{3}\right)^{\frac{1}{\rho}} \leq|z| \leq r\left[\frac{k}{h}\left(\frac{3}{\varepsilon}\right)^{\frac{1}{\rho}}\right] \frac{2}{3 \varepsilon \rho}
$$

un domaine $\mathcal{A}$, d'un seul tenant, tel que les arguments extrêmes, déterminés par continuité, des points de $\Delta$, diffèrent de plus de $\frac{\pi}{\varrho(\mathrm{I}+3 \varepsilon)}$.

L'épaisseur relative de la couronne circulaire $(I)$ reste finie si l'on donne à $\varepsilon$ une valeur fixe. Si l'on fait tendre $\varepsilon$ vers zéro, la différence des arguments extrêmes du domaine $\mathcal{A}$ tend vers $\frac{\pi}{\varrho}$, mais l'épaisseur relative de la couronne $(I)$ tend vers l'infini.

35. Nous appliquerons dans la suite le théorème XI principalement aux fonctions entières d'ordre supérieur à $\frac{\mathrm{I}}{2}$; l'application de ce théorème aux fonc29 - 2822. Acta mathematica. 52. Imprimé le 18 septembre 1928. 
tions entières d'ordre inférieur à $\frac{I}{2}$ permet de retrouver un théorème bien connu, de M. Wiman; nous allons même voir que ce théorème s'étend à certaines fonctions entières dont l'ordre est compris entre $\frac{\mathrm{I}}{2}$ et $\mathrm{I}$.

Considérons d'abord une fonction $F(z)$ dont les zéros $a_{1}, a_{2}, \ldots a_{n}, \ldots$ sont réels et positifs, et dont le développement en produit infini est de la forme:

$$
F(z)=C \prod_{n=1}^{\infty}\left(\mathrm{I}-\frac{z}{a_{n}}\right)
$$

L'ordre de cette fonction est au plus égal à un. En désignant par $V(r)$ le maximum de $\log |F(z)|$ sur le cercle $|z|=r$, on a:

$$
e^{V(r)}=|c| \prod_{n=1}^{\infty}\left(\mathrm{I}+\frac{r}{a_{n}}\right) .
$$

Supposons que pour une suite de valeurs: $r_{1}, r_{2}, \ldots r_{n}, \ldots$ tendant vers l'infini, on ait:

$$
\lim \frac{V\left(r_{n}\right)}{r_{n}^{\frac{1}{2}-\varepsilon^{\prime}}}=0
$$

$\varepsilon^{\prime}$ étant une constante positive.

Alors, d'après le théorème XI, il existe, à l'intérieur de la couronne circulaire:

$$
r \leq|z| \leq R
$$

un domaine $\Delta$ où la fonction $F(z)$ vérifie l'inégalité:

$$
\log |F(z)|>\frac{2}{3} \varepsilon^{\prime}|z|^{\frac{1}{2}-\varepsilon^{\prime}}
$$

et les arguments extrêmes de $\boldsymbol{A}$ diffèrent de plus de $2 \pi$ : le domaine $\boldsymbol{A}$ coupe la partie positive de l'axe réel.

Les quantités $r$ et $R$ sont respectivement inférieure et supérieure à $r_{n}$, et sont déterminées par les inégalités: 
(14)

$$
\left\{\begin{array}{l}
V(r) \leq \frac{\mathrm{I}}{3} r_{n}{ }^{\frac{1}{2}-\varepsilon^{\prime}} \\
V(R) \leq \frac{2}{9} \varepsilon^{\prime}\left(\frac{R}{r_{n}}\right)^{\varepsilon^{\prime}\left(\frac{1}{2}-\varepsilon^{\prime}\right)} R^{\frac{1}{2}-\varepsilon^{\prime}}
\end{array}\right.
$$

D'après la condition (I 2), on voit que l'on peut toujours choisir $R$ de façon' que la deuxième inégalité (I4) soit vérifiée. ${ }^{1}$

La fonction $F(z)$ étant une fonction à zéros réels et positifs, le minimum de son module sur le cercle $|z|=r$ a lieu sur la partie positive de l'axe réel, de sorte que l'inégalité (I3) étant vérifiée en une suite de points s'éloignant indéfiniment sur cet axe, elle est également vérifiée sur. une suite de circonférences, de centre o, s'éloignant indéfiniment.

36. On passe ensuite au cas d'une fonction entière dont le développement est de la forme:

$$
f(z)=C \prod_{n=1}^{\infty}\left(\mathrm{I}-\frac{z}{\alpha_{n}}\right)
$$

les $\alpha_{n}$, de modules $a_{n}$, ayant des arguments quelconques.

En désignant par $F(z)$ la fonction dont les zéros sont les $a_{n}$, si cette fonction satisfait à la condition $(\mathrm{I} 2)^{2}$, il existe une suite de cercles, s'éloignant indéfiniment, sur lesquels on a l'inégalité:

$$
\log |f(z)|>\frac{2}{3} \varepsilon^{\prime}|z|^{\frac{1}{2}-\varepsilon^{\prime}}
$$

En effet, sur l'un quelconque de ces cercles, on a:

$$
\min .|f(z)|>\min .|F(z)|
$$

${ }^{1}$ Il en est encore de même si, au lieu de considérer $\varepsilon^{\prime}$ comme une constante positive, on suppose que c'est une fonction de $r$ tendant vers zéro lorsque $r$ augmente indéfiniment, de façon $\varepsilon^{\prime}$ que $\varepsilon^{\prime} r^{2}$ augmente indéfiniment; on peut prendre par exemple:

$$
\frac{\mathrm{I}}{\varepsilon^{\prime}} \leq[\log r]^{1-\eta}
$$

$\eta$ étant une constante positive arbitrairement petite.

${ }^{2}$ Dans une note des Compte-Rendus, (23 janv. 1928, p. 213 ) j'avais cru pouvoir affirmer qu'il en est de même lorsqu'on remplace, dans la condition (12), la fonction $V(r)$ relative à $F(z)$ par la fonction analogue $V_{1}(r)$ relative à $f(z)$. Mais rien ne le prouve a priori. 
L’inégalité (I5) est en particulier vérifiée si l'ordre de la fonction $f(z)$ est inférieur à $\frac{\mathrm{I}}{2}$, car il en est de même pour la fonction $F(z)$, et l'inégalité (I2) est vérifiée quel que soit $r$. Nous retrouvons ainsi le théorème de M. Wiman ${ }^{1}$; la démonstration précédente est un peu plus précise que celle qui a été donnée par MM. Phragmén et Lindelöf, mais la méthode est analogue.

\section{CHAPITRE IV.}

Étude des cercles de remplissage des fonctions entières d'ordre supérieur à $\frac{1}{2}$.

37. Avant d'aborder l'étude des fonctions holomorphes dans des secteurs de couronnes circulaires, nous allons résoudre le problème suivant:

Etant donnée une fonction holomorphe $g(x)$ dans un domaine, et petite dans un cercle intérieur au domaine, on suppose que le maximum de $|g(x)|$ dans le cercle concentrique et de rayon double, est assez grand, et on se propose d'en déduire une limite inférieure du nombre des zéros de $g(x)-a$ dans un cerle concentrique et plus grand.

Pour fixer les idées, nous prendrons une fonction $g(x)$ holomorphe dans le cercle $|x|=30$. Le maximum du module de la fonction est supérieur ou égal à un dans (et sur) le cercle $|x|=\mathbf{r}$. Enfin, à l'intérieur du cercle $|x|=\frac{1}{2}$, la fonction $g(x)$ vérifie l'inégalité:

$$
\log |g(x)|<-\mu
$$

$\mu$ étant une quantité positive assez grande.

Il résulte des hypothèses précédentes, qu'en un certain point $x_{0}$ dont le module est compris entre $\frac{I}{2}$. et $I$, la fonction $g(x)$ et sa dérivée vérifient les inégalités:

$$
\begin{aligned}
& \left|g\left(x_{0}\right)\right| \leq \frac{\mathrm{I}}{2} \\
& \left|g^{\prime}\left(x_{0}\right)\right| \geq \mathrm{I} .
\end{aligned}
$$

${ }^{1}$ Wïman. Sur une extension d'un théorème de M. Hadamard (Arkiv för Math., Ast., Phys. t. II, $\mathrm{n}^{\circ} 14$ ) 
Sur toute circonférence de centre $x_{0}$, dont le rayon $\lambda$ est compris entre $\left|x_{0}\right|-\frac{1}{4}$ et $\left|x_{0}\right|+\frac{1}{4}$, il existe un arc de longeur $\frac{3}{4} \lambda$ sur lequel l'inégalité (i6) est vérifiée. En appliquant cette remarque à la première inégalité fondamentale de M. R. Nevanlinna, on en déduit l'inégalité:

$$
T\left(\left|x-x_{0}\right|=\lambda, g\right)>\frac{3}{26} \mu
$$

dès que $\mu$ dépasse une constante numérique. On peut aussi écrire:

$$
T\left(\left|x-x_{0}\right|=\lambda, g-2\right)>\frac{\mathrm{I}}{9} \mu
$$

Désignons par $n$ le plus grand des nombres des zéros de $g(x)$ et de $g(x)$ - I situés dans le cercle de centre $x_{0}$ et de rajon $\left|x_{0}\right|+\frac{\mathrm{I}}{2}$ et par $n^{\prime}$ le plus grand des nombres des zéros de $g(x)-2$ et de $g(x)-3$ situés dans le même cercle.

D'aprés le théorème de Boutroux, on peut choisir une quantité $R$ comprise entre $\left|x_{0}\right|+\frac{1}{4}$ et $\left|x_{0}\right|+\frac{1}{2}$, de façon que l'on ait:

$$
\begin{array}{ll}
N\left(\left|x-x_{0}\right|=R, \alpha\right)<5 n & (\alpha=0, \mathrm{I}) \\
N\left(\left|x-x_{0}\right|=R, \beta\right)<5 n^{\prime} & (\beta=2,3) .
\end{array}
$$

En appliquant l'inégalite (5) aux fonctions $g(x)$ et $g(x)-2$, et faisant, dans cette inégalité, $r=\left|x_{0}\right|-\frac{\mathrm{I}}{4}$, on constate que $n$ et $n^{\prime}$ dépassent nécessairement $\frac{\mathrm{I}}{2400} \mu$.

Ensuite l'application du corollaire du théorème I fournit une limite inférieure du nombre des zéros de $g(x)-a$ situés dans le cercle de centre $x_{0}$ et de rayon $20\left(\left|x_{0}\right|+\frac{I}{2}\right)$, cercle qui est contenu dans le cercle $|x|=30$. Cette limite inférieure est précisée dans l'énoncé suivant:

XIII. Soit $g(x)$ une fonction holomorphe dans le cercle $|x|=3$ o. On suppose que le maximum de $|g(x)|$ n'est pas inferieur à un dans le cercle $|x|=\mathrm{I}$, et qu'à l'intérieur du cercle $|x|=\frac{\mathrm{I}}{2}$, la fonction $g(x)$ vérifie l'inégalité:

$$
\log |g(x)|<-\mu
$$

$\mu$ dépassant une constante numérique positive. 
Alors le nombre des zéros de $g(x)-a$ situés dans le cercle $|x|=30$ est supérieur $\grave{a}$ $\frac{\mu}{\text { I } 700000}$, sauf peut-être pour des valeurs de a dont les représentations sphériques sont incluses dans deux petits cercles de rayon $e^{-\frac{\mu}{2400}}$, l'un d'eux entourant nécessairement le point de la sphère qui correspond au point à l'infini du plan des a.

38. Il serait interessant de rechercher, par une méthode de représentation conforme, qu'a employée M. Valiron dans un problème analogue ${ }^{1}$, une limite inférieure (fonction de $\varepsilon$ ) du nombre des zéros de $g(x)-a$ situés dans le cercle $|x|=\mathrm{I}+\varepsilon$.

39. Abordons l'étude des fonctions holomorphes dans certains secteurs de couronnes circulaires.

Rappelons d'abord une inégalité due à M. Valiron ${ }^{2}$ :

Soit $g(x)$ une fonction holomorphe, et ne prenant pas les valeurs zéro et un dans le cercle $|x|=\mathrm{I}$. Dès que $|g(\mathrm{o})|$ dépasse une constante numérique, la fonction $g(x)$ vérifie l'inégalité:

$$
\log |g(x)|<\frac{1-|x|}{3} \log |g(0)|
$$

Ceci posé, considérons une fonction $f(z)$ holomorphe et ne prenant pas les valeurs zéro et un dans le secteur $(S)$ de couronne circuläire déterminé par les inégalites:

$$
\begin{gathered}
-\frac{\pi}{2} \leq \operatorname{Arg} z \leq+\frac{\pi}{2} \\
r_{1} \leq|z| \leq r_{2}
\end{gathered}
$$

avec:

$$
r_{2}>4 r_{1} \text {. }
$$

Soit $M$ un point de la circonférence:

$$
|z|=r^{\prime}=\sqrt{r_{1} r_{2}}
$$

tel que le plus petit are de cette circonférence, limité d'une part en $M$, d'autre part au contour rectiligne du secteur $(S)$, ait pour longueur $\beta r$.

1 G. Valiron. Sur quelques propriétés des fonctions méromorphes (Compte-Rendus, t. I86, 2 avril 1928, p. 935).

${ }^{2}$ G. Valiron. Compléments au théorème de Picard-Julia, cité plus haut. Voir aussi la thèse de M. David R. Williams: Compléments au théorème de M. Julia (Rendiconti del Circolo Matematico di Palermo, t. LII, 1928). 
Considérons, s'il y a lieu, une suite de circonférences tangentes an contour rectiligne $d u$ secteur $(S)$, puis cinq circonférences de rayon $\frac{r}{2}$, toutes ces circonférences ayant leurs centres à la distance $r$ de l'origine, et telles que chacune d'elles a son centre sur la circonférence concentrique à la précédente et de rayon moitié; appliquons chaque fois l'inégalité (I9), en faisant $|x|=\frac{\mathrm{I}}{2}$; nous aboutissons à l'inégalité suivante:

$$
\log |f(P)|>\left(\frac{\beta}{4}\right)^{5} \log |f(M)|
$$

$P$ désigne le point de l'axe réel, situé dans le secteur $(S)$, à la distance $r$ de l'origine.

L'inégalité (20) est valable dès que son deuxième membre dépasse une constante numérique.

De l'inégalité (2o), nous allons déduire, par une nouvelle application de l'inégalité (19), une limite inférieure de $\log |f(z)|$ sur le segment de l'axe réel et positif dont les points sont situés à une distance de l'origine comprise entre $2 r_{1}$ et $\frac{r_{2}}{2}$.

Il suffit de construire la circonférence de centre $P$ et de rayon $r-r_{1}$, et d'appliquer l'inégalité (19) en faisant:

$$
\mathrm{I}-|x|=\frac{r_{1}}{r-r_{1}}
$$

puis en recommençant, après la transformation:

$$
Z=\frac{r^{2}}{z}
$$

ce qui conduit à l'énoncé suivant:

XIV. Soit $f(z)$ une fonction holomorphe et ne prenant pas les valeurs zéro et un dans un secteur $(S)$, d'ouvrture $\pi$, de la couronne circulaire limitée par les cir. conférences de centre o et de rayons $r_{1}$ et $r_{2}$. Soit $M$ un point du secteur $(S)$, situé sur. la circonférence $|z|=r=\sqrt{r_{1} r_{2}}$; le plis petit are de cette circonférence, limité en $M$ et au contour du secteur $(S)$, a une longueur désignée par $\beta r$.

Ceci posé, sur la partie de la bissectrice du secteur (S), satisfaisant aux inégalités: 


$$
2 r_{1} \leq|z| \leq \frac{1}{2} r_{2}
$$

la fonction $f(z)$ vérifie l'inégalité:

$$
\log |f(z)|>\frac{\mathrm{I}}{3}\left(\frac{\beta}{4}\right)^{5} \frac{r_{1}}{r} \log |f(M)| .
$$

Les conditions d'application de cette proposition sont les suivantes:

$$
\begin{gathered}
r_{2}>4 r_{1} \\
\left(\frac{\beta}{4}\right)^{5} \log |f(M)|>C
\end{gathered}
$$

$C$ désigne une constante numérique.

Remarque. Une étude analogue à l'étude précédente permet de fixer une limite inférieure de $|f(z)|$ non plus seulement sur une partie de la bissectrice du secteur, mais encore sur des segments situés à l'intérieur, sur des rayons issus de l'origine.

40. Si l'inégalité (20) n'est pas vérifiée, la fonction $f(z)$ prend nécessairement la valeur zéro ou la valeur un dans le secteur $(S)$. Nous allons montrer que si la fonction $f(z)$ est holomorphe dans le secteur $\left(S_{1}\right)$, d'ouverture $\pi(\mathrm{I}+\alpha)$, de la couronne circulaire limitée par les circonférences de centre o et de rayons $\frac{r_{1}}{2}$ et $2 r_{2}$, les secteurs $\left(S_{1}\right)$ et $(S)$ possédant même bissectrice, il existe (moyennant une hypothèse supplémentaire qui sera précisée dans la suite), dans le secteur $\left(S_{1}\right)$, un cercle de remplissage à l'intérieur duquel le nombre des zéros de $f(z)-a$. est, sauf pour des valeurs exceptionnelles de $a$, de l'ordre de grandeur de log $|f(M)|$

Nous désignerons par $\left(S_{2}\right)$ le secteur, d'ouverture $\pi\left(1+\frac{\alpha}{30}\right)$ de la couronne circulaire limitée par les circonférences de centre o et de rayons $\frac{2}{3} r_{1}$ et $\frac{3}{2} r_{2}$, et ayant même bissectrice que les secteurs $(S)$ et $\left(S_{1}\right)$.

Nous désignerons par $V(R)$ le maximum de $\log |f(z)|$ sur la partie de la circonférence $|z|=R$, intérieure au secteur $(S)$.

Nous distinguerons deux cas:

Premier cas. Il existe un nombre a de module inférieur à un, tel que le nombre des zéros de $f(z)$-a intérieurs au secteur. $\left(S_{2}\right)$ est inférieur à un nombre $N$ précisé dans la suite. 
Quitte à opérer une translation des valeurs de $f(z)$, nous supposerons que $a$ est nul, et nous désignerons par $a_{1}, a_{2}, \ldots a_{n}, \ldots$ les zéros de $f(z)$ intérieurs au secteur $\left(S_{2}\right)$. Nous allons faire l'étude de la fonction:

$$
\varphi(z)=\frac{f(z)}{\prod\left(\mathrm{I}-\frac{z}{a_{n}}\right)} e^{5 N \log \frac{r}{r_{1}}}
$$

Cette fonction est holomorphe dans le secteur $\left(S_{2}\right)$ et n'y possède plus de zéros. Au point $M$ situé sur la circonférence $|z|=r$, en une position précisée dans l'énoncé XIV, la fonction $\varphi(z)$ vérifie l'inégalité:

$$
\log |\varphi(M)|>\log |f(M)|
$$

puisque:

$$
\prod\left|\mathrm{I}-\frac{z}{a_{n}}\right|<e^{\frac{5}{2} N \log \frac{r}{r_{1}}}
$$

(On suppose que $\frac{r}{r_{1}}$ dépasse une constante numérique).

Recherchons maintenant une limite inférieure de $\log |\varphi(z)|$ sur un arc de cercle voisin de l'arc de cercle $|z|=r_{1}$ intérieur au secteur $\left(S_{2}\right)$. D'après le théorème de Boutroux, il existe une circonférence de centre o et de rayon $r_{1}^{\prime}$ compris entre $r_{1}$ et $2 r_{1}$, telle qu'en tout point $z$ de cette circonférence, on a l'inégalité:

$$
\prod\left|\mathrm{I}-\frac{z}{a_{n}}\right|>e^{-3 N}
$$

ce qui entraîne l'inégalité:

$$
\log |\varphi(z)|<V\left(r_{1}{ }^{\prime}\right)+6 N \log \frac{r}{r_{1}}
$$

en tout point $z$ du secteur $(S)$, ayant pour module $r_{1}^{\prime}$.

Supposons maintenant vérifiée l'inégalité:

$$
V\left(r_{1}{ }^{\prime}\right)+6 N \log \frac{r}{r_{1}} \leq \frac{\mathbf{I}}{3}\left(\frac{\beta}{4}\right)^{5} \frac{r_{1}}{r} \log |\varphi(M)|
$$

de manière à pouvoir appliquer la réciproque du théorème XIV.

30-- 2822. Acta mathematica. 52. Imprimé le 19 septembre 1928. 
Nous allons séparer l'inégalités (24) en deux autres inégalités en formulant les deux hypothèses supplémentaires suivantes:

$$
N=\frac{\mathrm{I}}{80}\left(\frac{\beta}{4}\right)^{5} \frac{r_{1}}{r} \log |f(M)| \frac{\mathrm{I}}{\log \frac{r}{r_{1}}}
$$

$$
V\left(\lambda r_{1}\right) \leq \frac{\mathrm{I}}{4}\left(\frac{\beta}{4}\right)^{5} \frac{r_{1}}{r} \log |f(M)| \quad(\mathrm{I}<\lambda<2)
$$

L'ensemble de ces deux inégalités entraîne l'inégalité (24) en vertu de. l'inégalité (22).

$\lambda$ étant un nombre quelconque compris entre $I$ et 2 , on peut, dans l'inégalité (26), remplacer $\lambda r_{1}$ par $r_{1}^{\prime}$.

Ceci posé, il résulte de la réciproque du théorème XIV que la fonction $\varphi(z)$ prend nécessairement la valeur un en un point $P$ intérieur au secteur $(S)$; comme cette fonction est dépourvue de zéros dans le secteur $\left(S_{2}\right)$, son module ne dépasse pas un sur une courbe issue de $P$ et traversant le secteur $\left(S_{1}\right)$. Par suite, sur cette courbe la fonction $f(z)$ vérifie l'inégalité:

$$
\log |f(z)| \leq-\frac{5}{2} N \log \frac{r}{r_{1}}
$$

Traçons une suite de circonférences dont les centres vont de $P$ à $M$ à l'intérieur du secteur $(S)$, et dont les rapports des rayons aux distances de leurs centres à l'origine sont égaux à $\frac{\pi}{30} \alpha$, de façon que ces circonférences soient intérieures au secteur $\left(S_{2}\right)$. De plus, le centre d'une circonférence quelconque de la suite est situé sur la circonférence concentrique à la précédente et de rayon moitié. Le nombre total de ces circonférences peut être pris inférieur au nombre $p$ déterminé par l'équation:

$$
\left(\mathrm{I}+\frac{\pi \alpha \alpha}{30}\right)^{p}=2 \frac{r}{r_{1}}
$$

ou encore inférieur au nombre $\frac{\text { IO }}{\alpha} \log \frac{r}{r_{1}}$. 
D'après un théorème résultant d'une inégalité de $\mathbf{M}$. Carleman ${ }^{1}$, si la fonction $f(z)$ était inférieure en module à un dans toutes ces circonférences, on en déduirait l'inégalité:

$$
\log |f(M)|<-\frac{5}{2} N\left(\frac{r_{1}}{r^{\alpha}}\right)^{\frac{400}{\alpha}} \log \frac{r}{r_{1}} .
$$

L'inégalité (27) étant en contradiction avec la valeur de $|f(M)|$, il existe nécessairement une circonférence $(C)$, que nous supposerons la première rencontrée à partir du point $P$, telle que le maximum du module de la fonction $f(z)$ dans ce cercle est supérieur ou égal ì un. Le raisonnement qui a conduit à l'inégalité (27) s'applique d'ailleurs à la suite des circonférences qui précèdènt la circonférence $(C)$, de sorte que l'inégalité (27) est vérifiée sur une courbe issue $d u$ centre du cercle $(C)$. On pourra réduire le rayon du cercle $(C)$, en conservant le centre, jusqu'à une valeur telle que dans le cercle $\left(C^{\prime}\right)$ ainsi construit, $|f(z)|$ soit inférieur à un, le maximum de ce module étant égal à un $s u$. la circonférence $\left(C^{\prime}\right)$. Dans le cercle concentrique au cercle $\left(C^{\prime}\right)$ et de rayon moitié, la fonction $f(z)$ satisfait à l'inégalité (27), dont noùs désignerons le deuxième membre par - $\mu$.

Nous sommes dans les conditions d'application du théorème XIII, qui fixe une limite inférieure du nombre des zéros de $f(z)-a$ (sauf pour des valeurs exceptionnelles de $a$ ) dans le cercle $\left(C^{\prime \prime}\right)$ concentrique à $\left(C^{\prime}\right)$ et de rayon 30 fois plus grand. Ce dernier rayon est inférieur à $\pi \alpha|z|, z$ désignant l'affixe du centre de $\left(C^{\prime \prime}\right)$, et, par suite, le cercle $\left(C^{\prime \prime}\right)$ se trouve tout entier situé dans le secteur $\left(S_{1}\right)$.

Deuxième cas. Dans le secteur $\left(S_{2}\right)$, le nombre des racines de toute équation $f(z)-a=0$ est, pour a inférieur en module à un, supérieur au nombre $N$ déterminé par l'égalité (25).

On applique le théorème II au secteur $\left(S_{1}\right)$ en procédant à la division de ce secteur en $p$ domaines partiels inclus dans des cercles tels que le rapport de

${ }^{1}$ Voir H. Milloux. Le théorème de M. Picard,... (cité plus haut). Le théorème que nons utilisons ici est le suivant: Si une fonction $g(x)$ est holomorphe et inférieure en module à un dans le cercle $|x|=I$, et si elle est inférieure en module à $m$ sur un are de courbe issu du centre et traversant ce cercle, elle vérifie, dans le cercle $|x|=\frac{I}{2}$, l'inégalité:

$$
|g(x)|<m^{k}
$$

$k$ étant une certaine constante numérique. 
leurs rayons à la distance de leurs centres à l'origine soit égale à $\frac{\pi \alpha}{30}$, comme pour les cercles $\left(C^{\prime}\right)$. Un calcul simple établit que le nombre $p$ est inférieur à

$$
\frac{600}{\alpha^{2}} \log \frac{v}{r_{1}}
$$

Il existe donc un cercle $\left(C^{\prime \prime}\right)$ analogue au cercle $\left(C^{\prime \prime}\right)$ du premier cas, dans lequel le nombre des zéros de $f(z)-a$ est, sauf pour des valeurs exceptionnelles de $a$, supérieur à:

$$
\frac{N \alpha^{2}}{420000 \log \frac{r}{r_{1}}}
$$

4I. On passe ensuite à l'étude d'une fonction holomorphe dans un secteur $\left(S_{2}\right)$ d'ouverture $\frac{\pi}{\varrho^{\prime}}(\mathrm{I}+\alpha)$, par la transformation conforme: $Z=z^{0^{\prime}}$.

La transcription des résultats obtenus dans le premier et le deuxième cas fournit le théorème suivant:

$\mathrm{XV}$. Soit $f(z)$ une fonction holomorphe dans un sectew $(S)$, d'ouverture $\frac{\pi}{\varrho^{\prime}}(\mathrm{I}+\alpha)$, de la couronne circulaire dont les points ont leurs modules compris entre $r_{1}$ et $r_{2}$. On désigne par $V(R)$ le maximum de log $|f(z)|$ sur la partie de la circonférence $|z|=R$ intérieure au secteur $(S)$. Soit $M$ un point, de module $r=\sqrt{r_{1} r_{2}}$, intérieur au secteur $(S)$. Désignons par $\frac{r}{\varrho^{\prime}}(\pi \alpha+\beta)$, ( $\beta$ étant, de même que $\alpha$, une constante positive), une limite inférienve de la longueur du plus petit arc de la circonfërence $|z|=r$, limité en $\boldsymbol{M}$ et au contour de $(S)$.

Si l'inégalité:

$$
V\left(\lambda r_{1}\right)<\frac{\mathrm{I}}{\mathrm{IO}^{4}} \beta^{5}\left(\frac{r_{1}}{r}\right)^{e^{\prime}} \log |f(M)|
$$

est vérifiée, quel que soit le nombre $\lambda$ compris entre $2^{\frac{1}{\rho^{\prime}}}$ et $4^{\frac{1}{\rho^{\prime}}}$, il existe, à l'intérieur du secteur $(S)$, un cercle de remplissage, vu de l'origine sous l'angle $\frac{x \alpha}{\varrho^{\prime}}$, à l'intérieur. duquel le nombre des zéros de $f(z)-$ a est supérieur à :

$$
n=\frac{\mathrm{I}}{\mathrm{IO}^{11}} \beta^{5}\left(\frac{r_{1}}{r}\right)^{\frac{500}{\alpha} \rho^{\prime}} \log |f(M)|
$$


sauf peutêtre pour des valeurs de: a dont les représentations sphériques sont incluses dans deux petits cercles de rayon $e^{-n}$, l'un de ces cercles entourant le point de la sphère qui correspond au point à l'infini du plan des a.

Ce théorème est valable dès que $\left(\frac{r_{1}}{r}\right)^{e^{\prime}}$ dépasse une constante numérique.

42. Le théorème $\mathrm{XV}$ est applicable aux fonctions entières. Soit $f(z)$ une telle fonction; désignons par $V(R)$ le maximum de $\log |f(z)|$, non plus sur un arc du cercle, mais sur le cercle tout entier: $|z|=R$. Il sera toujours possible, étant donnée une valeur quelconque $r_{1}$, de déterminer une valeur $r$ telle que l'inégalité (28) soit vérifiée, lorsque l'ordre de la fonction entière $f(z)$ dépasse $\varrho^{\prime}$.

Appliquons aux fonctions entières $f(z)$ d'ordre fini $\varrho$ supérieur à $\frac{1}{2}$. Les inégalités:

$$
\begin{aligned}
& \log |f(z)|<r^{0}[1+\varepsilon(r)] \\
& \log |f(z)|>r^{0[1-\varepsilon(r)]}
\end{aligned}
$$

sont vérifiées, la première à partir d'une certaine valeur de $r=|z|$, la deuxième pour une suite de valeurs de $r$ tendant vers l'infini; $\varepsilon(r)$ est une fonction de $r$ tendant vers zéro lorsque $r$ augmente indéfiniment. Le choix de cette fonction comporte un certain arbitraire; on pourra supposer que cette fonction $\varepsilon(r)$. est une fonction constamment décroissante, et, quitte à examiner plus tard un important cas particulier, telle que $\varepsilon(r) \log r$ tend vers l'infini avec $r$.

Ceci posé, nous prendrons comme point $M$ un point en lequel la fonction $f(z)$ vérifie l'inégalité:

$$
\log |f(M)|>\beta r^{\varrho[1-\varepsilon(r)]}
$$

$r$ désigne le module de l'affixe de $\boldsymbol{M}$, et $\beta$ une constante inférieure à un, pour le moment indéterminée. L’inégalité:

$$
\left(\frac{r_{1}}{r}\right)^{\rho\left[1+\varepsilon\left(r_{1}\right)\right]-\varrho^{\prime}}<\frac{\beta^{6}}{40000} r^{-2 \rho \varepsilon\left(r_{1}\right)}
$$

entraîne l'inégalité (28).

Choisissons:

$$
\begin{gathered}
\varrho^{\prime}=\varrho\left[\mathrm{I}-\sqrt{\varepsilon\left(r_{1}\right)}\right] \\
r_{1}^{1+10} \sqrt{\varepsilon\left(r_{1}\right)}=r
\end{gathered}
$$


et, pour valeur de $\beta$, la plus grande des deux quantités:

$$
r=Q \varepsilon(r) \text { et } \sqrt{\varepsilon(r)} \text {. }
$$

Il résulte de ce choix que l'inégalité $\left(28^{\prime}\right)$, et par suite l'inégalité (28), sont vérifiées dès que $r^{e^{\varepsilon(r)}}$ dépasse une constante numérique.

Enfin, nous achèverons l'application du théorème $\mathrm{XV}$ en choississant pour valeur de $\alpha$ :

$$
\alpha=500\left[\varepsilon\left(r_{1}\right)\right]^{\frac{1}{4}}
$$

et le nombre $n$ précisé dans ce théorème est supérieur à:

$$
\left.r^{[1-10} \sqrt[4]{\varepsilon\left(r_{1}\right)}\right]
$$

43. Nous avons fait l'application du théorème $\mathrm{XV}$ en partant d'un point $M$ en lequel l'inégalité (29) est vérifiée. D'après le théorème XI, on peut prendre arbitrairement ce point $M$ dans un domaine dont l'extension angulaire est voisine de $\frac{\pi}{\varrho}$. Il suffit de remplacer, dans ce théorème XI, les quantités $\varepsilon$ et $\varrho^{\prime}$ respectivement par $\beta$ et $\varrho[\mathrm{I}-\varepsilon(r)]$. L'extension angulaire du domaine des points $M$ est alors supérieure à $\frac{\pi}{\varrho[\mathrm{I}+4 \beta]}$, et les modules des points $M$ sont compris entre les nombres $r_{1}^{\prime}$ et $r_{2}^{\prime}$ vérifiant les inégalités:

$$
r_{1}^{\prime} \varrho\left[1+\varepsilon\left(r_{1}{ }^{\prime}\right)\right] \leq \frac{1}{3} r^{\circ}[1-\varepsilon(r)]
$$

et

$$
r_{2}^{\prime} \varrho\left[1+\varepsilon\left(r_{2}^{\prime}\right)\right] \leq \frac{\beta}{3} r_{2}{ }^{\prime} \varrho[1-\varepsilon(r)]\left(\frac{r_{2}^{\prime}}{r^{*}}\right)^{\frac{3 \beta}{2} \rho[1-8(r)]}
$$

On pourra prendre par exemple:

$$
\begin{aligned}
r & =r_{1}{ }^{\prime} 1+3 \varepsilon\left(r_{1}^{\prime}\right) \\
r_{2}^{\prime} & =r^{1+3} \sqrt{\varepsilon(r)} .
\end{aligned}
$$

Rappelons que le domaine des points $M$ contient, sur la circonférence $|z|=r$, un point en lequel on a:

$$
\log |f(z)|>r^{\bullet}[1-\varepsilon(r)]
$$


En appliquant les résultats précédents à chacun des points $M$ du domaine, on obtient le théorème suivant:

XVI. Soit $f(z)$ une fonction entière d'ordre $\varrho$, supérieur $\grave{a} \frac{\mathrm{I}}{2}$, et soit $z$ un point de module $r$, tel que:

$$
\log |f(z)|>\vartheta^{Q}[1-\varepsilon(1)]
$$

$\varepsilon(t)$ étant une fonction tendant vers zéro, en décroissant constamment, lorsque $t$ augmente indéfiniment.

Soit $S(\omega)$ les secteurs de couronnes circulaires, limités par les cercles de centre o et de rayons:

$$
\begin{aligned}
& r_{1}=r^{1-i(r)} \\
& r_{2}=r^{.1+\gamma(r)}
\end{aligned}
$$

et par des segments faisants entre eux l'angle $\frac{\pi}{\varrho}[\mathrm{I}+\gamma(\vartheta)]$. w désigne l'angle polaire de la bissectrice du secteur: $S(\omega)$.

Ceci posé, à l'intérieur de chaque secteur $S(\omega)$ correspondant à une suite continue de valeurs de $\omega$, contenant l'argument du point $z$, et dont les valeurs limites diffèrent de plus de:

$$
\frac{2 \pi}{\varrho}\left[\mathrm{I}-\gamma^{\prime}(r)\right]
$$

il existe un point, centre d'un cercle de remplissage, vu de l'origine sous un angle égal à $\frac{\pi}{\varrho} \delta(r)$, à l'intérieur duquel le nombre des zéros de $f(z)-a$ est supérieur à

$$
n=r^{o}\left[1-\frac{\gamma(r)}{\delta(r)}\right]
$$

sauf peut-être pour des valeurs de a dont les représentations sphériques sont incluses dans deux petits cercles de rayon $e^{-n}$, l'un de ces cercles entourant le point de la sphère qui correspond au point à l'infini du plan des a.

$\gamma(t)$ et $\gamma^{\prime}(t)$ sont des fonctions tendant vers zéro avec $\frac{\mathrm{I}}{t}$, et dépendent du choix de la fonction $\varepsilon(t)$. Leurs expressions résultent de l'étude précédente. En particulier, si la décroissance de la fonction $\varepsilon(t)$ n'est pas trop rapide (ce que l'on peut toujours supposer, puisque l'on peut augmenter arbitrairement cette fonction), on peut prendre pour $\gamma(t)$ une certaine puissance positive de $\varepsilon(t)$. 
Quant à la fonction $\delta(t)$, son choix est arbitraire, mais il est tout indiqué de le prendre tel que le rapport $\frac{\gamma(t)}{\delta(t)}$ tende vers zéro avec $\frac{\mathrm{I}}{t}$, de façon que le nombre des zéros de $f(z)$ - a situés dans chaque cercle de remplissage soit de l'ordre de $r^{e}$.

D'ailleurs, si l'on voulait les cercles de remplissage dont les rayons soient les plus petits possible, on pourrait utiliser la méthode suivante: prendre $\delta(t)$ constant, et appliquer à un cercle de remplissage déduit du théorème XVI les résultats des théorèmes IX et $\mathrm{X}$, la valeur exceptionnelle de la fonction étant ici la valeur. $\infty$.

44. Des résultats plus précis peuvent être obtenus lorsque la fonction $f(z)$ est une fonction entière à croissance très régulière, dont la fonction $V(r)$ satisfait aux deux inégalités:

$$
(h r)^{\circ}<V(r)<(k r)^{\circ}
$$

$h$ et $k$ désignant deux constantes positives.

Sur chaque circonférence $|z|=r$, il existe un point, d'argument $\omega_{0}$, en lequel la fonction $f(z)$ vérifie l'inégalité:

$$
\log |f(z)|>(h r) \text {. }
$$

Ce point peut être pris pour centre d'un secteur $S\left(\omega_{0}\right)$ d'ouverture angulaire $\frac{\pi}{\varrho}(\mathrm{I}+\alpha)$, et d'épaisseur relative finie, puisque l'inégalité $(28)$ est vérifiée $\left(\beta=\frac{\pi}{2}\right)$ dès que $\frac{r_{1}}{r}$ est inférieur à une constante dépendant uniquement de $h$ et $k$. Dans ce secteur $S\left(\omega_{0}\right)$, il existe un cercle de remplissage, vu de l'origine sous l'angle $\frac{\pi}{\varrho} \delta(r)$, et à l'intérieur duquel le nombre des zéros de $f(z)$-a est supérieur à:

$$
n=r^{\rho} e^{-\frac{k^{\prime}}{\delta(r)}}
$$

$k^{\prime}$ étant une constante dépendant uniquement de la fonction $f(z)$; et ceci pour toutes les valeurs de $a$, sauf pour des valeurs exceptionnelles à représentations sphériques incluses dans deux petits cercles de rayon $e^{-n}$, l'un de ces cercles entourant le point de la sphère qui correspond à l'infini.

On peut également prendre comme centre du secteur $S(\omega)$ l'un des points $M$ du domaine dont l'existence est établie au théorème XII, et même construire 
le secteur $S(\omega)$ de façon que le point $M$ reste à la distance $\beta r$ du contour de ce secteur. Tant que $\beta$ demeure supérieur à une constante positive $\varepsilon$, et que l'argument de $M$ varie entre deux limites $\omega_{1}$ et $\omega_{2}$ dont la différence est inférieure à $\frac{\pi}{\varrho}-\varepsilon$, et qui sont précisées dans l'énoncé XII, le secteur $S(\omega)$ a une épaisseur relative finie. Mais cette épaisseur tend vers l'infini quand $\varepsilon$ tend vers zéro.

45. Revenons au cas général, étudié au théorème XVI, et développons quelques conséquences de ce théorème:

Les cercles de remplissage dont l'existence est établie au théorème XVI sont générateurs de courbes de Borel, en ce sens que dans une suite quelconque de cercles de remplissage s'éloignant indéfniment, l'exposant de convergence des zéros de $f(z)-a$ est égal à $\varrho$, sauf pour une valeur au plus de a.

Ces cercles sont vus de l'origine sous des angles qui tendent vers zéro lorsque les cercles s'éloignent indéfiniment. Les arguments curvilignes des arguments des centres de ces cercles définissent des courbes de Borel ${ }^{1}$.

Bornons-nous à l'étude des arguments rectilignes limites de ceux des centres des cercles de remplissage. Il résulte de la position de ces cercles, position qui a été précisée dans le théoréme XVI, qu'en désignant par $\Omega$ le plus petit angle qui contient tous les arguments limites, l'angle $\Omega$ est supérieur ou égal au plus petit des nombres $\frac{\pi}{\varrho}, 2 \pi-\frac{\pi}{\varrho}$, d'où le théorème suivant:

XVII. Soit $\Omega$ le plus petit angle contenant à son intérieur (côtes compris) toutes les demi-droites de Borel d'une fonction entière d'ordre fini o supérieur à $\frac{\mathrm{I}}{2}$. L'ouverture de l'angle $\Omega$ ne peut être inférieure au plus petit des nombres $\frac{\pi}{\varrho}, 2 \pi-\frac{\pi}{\varrho}$.

Ce théoréme, qui précise un théorème dû à $\mathrm{M}$. Bieberbach ${ }^{2}$, a déjà été donné par M. Valiron ${ }^{3}$ (avec un énoncé un peu moins précis). Auparavant, je l'avais démontré pour les demi-droites de Julia de la fonction $f(z) .{ }^{4}$

\footnotetext{
1 Voir la signification au numéro I9.

${ }^{2}$ Bieberbach. Ueber eine Vertiefung des Picardschen Satzes bei gunzen Funktionen endlicher Ordnung (Math. Zeit. Bd. 3, I919, p. I75).

${ }^{8}$ G. Valiron. Recherches sur le théorème de M. Borel dans la théorie des fonctions méromorphies (déjà cité).

${ }^{4}$ H. Milloux. Sur la théorie des fonctions entières d'ordre fini (C. R. t. I85, I927, p. 1436$)$.

31-2822. Acta mathematica. 52. Imprimé le 19 septembre 1928.
} 


\section{CHAPITRE V.}

\section{Étude de certaines fonctions entières d'ordre fini supérieur a un.}

46. Une conséquence immédiate $d u$ théorème XVII est la suivante: Si une fonction entière $f(z)$ d'ordre fini $\varrho$ supérieur à un ne possède que deux demidroites de Borel, l'angle de ces deux demi-droites est égal à $\frac{\pi^{1}}{\varrho}$.

Dans ce chapitre, nous étudions certaines de ces fonctions.

Quitte à faire une rotation, on peut supposer que les arguments des deux demi-droites de Borel $O A$ et $O A^{\prime}$ sont égaux respectivement à $+\frac{\pi}{2 \varrho}$ et $-\frac{\pi}{2 \varrho}$.

Dans tout angle $B O B^{\prime}$ complètement intérieur à l'angle $A O A^{\prime}$ (dont l'ouverture est égale à $\left.2 \pi-\frac{\pi}{\varrho}\right)$, l'ordre $\varrho^{\prime}$ de la fonction $f(z)$ est inférieur à $\varrho$, sans quoi l'on en déduirait, d'après le théorème XVI, une suite, s'éloignant indéfiniment, de cercles de remplissage générateurs de courbes de Borel complètement intérieures à l'angle $A O A^{\prime}$, ce qui est contraire à l'hypothèse.

L'ordre $\varrho^{\prime}$ de la fonction $f(z)$ dans l'angle $B O B^{\prime}$ dépend de cet angle, et peut tendre vers $\varrho$ lorsque l'angle $B O B^{\prime}$ tend vers l'angle $A O A^{\prime}$. Nous limiterons l'étude des fonctions ne possédant que deux demi-droites de Borel aux fonctions $f(z)$ telles que l'ordre $\varrho^{\prime}$ de la fonction $f(z)$ dans tout angle completement intérieur à l'angle $A O A^{\prime}$ est inférieur à un nombre fixe inférieur à $\varrho$.

47. Tel est le cas des fonctions ne possédant que deux demi-droites de Julia. En effet, dans tout angle $B O B^{\prime}$, l'ordre de la fonction $f(z)$ ne pourrait dépasser $\frac{\varrho}{2 \varrho-I}$, sans qu'on en déduise, d'après le théorème XVI, l'existence d'une suite de cercles de remplissage, s'éloignant indéfiniment, et générateurs d'au moins une demi-droite de Julia intérieure à l'angle $A O A^{\prime}$; on pourrait même affirmer, dans cette hypothèse, l'existence d'une demi-droite $O D$ (intérieure à l'angle $A O A^{\prime}$ ) telle que dans tout angle d'ouverture arbitrairement petite, admettant pour bis-

1 La proposition est encore vraie, d'après le théorème XVII, pour $\varrho=I$; elle ne l'est plus lorsque $\varrho$ est inférieur à un, comme l'a montré $M$. Valiron dans son mémoire précédemment cité. 
sectrice $O D$, l'exposant de convergence des zéros de $f(z)-a$ serait supérieur à $\frac{\varrho}{2 \varrho-I}$, sauf pour une valeur au plus de $a$.

48. Reprenons l'étude de la fonction $f(z)$ ne possédant que deux demidroites de Borel, et satisfaisant à la condition restrictive précisée à la fin du numéro 46.

Si petite que soit la constante positive $\varepsilon$, la fonction:

$$
\varphi(z)=f(z) e^{-z^{\varrho(1-\varepsilon)}}
$$

tend vers zéro sur les deux rayons $O C$ et $O C^{\prime}$ d'argaments $\pm \frac{\pi}{2\left(\varrho-\frac{\varepsilon}{2}\right)}$, tandis que, si grande que soit la constante positive $A$, on peut toujours trouver, à l'intérieur de l'angle $C^{\prime} O C$, un point $M$ en lequel le module de la fonction $\varphi(z)$ surpasse $A$ : en effet, la fonction $f(z)$, et par suite la fonction $\varphi(z)$, sont holomorphes et d'ordre $\varrho$ à l'intérieur de l'angle $C^{\prime} O C$.

Donc, sur une courbe issue de $M$ et s'éloignant à l'infini nécessairement à l'intérieur de l'angle $C^{\prime} O C$, la fonction $\varphi(z)$ est supérieure en module à $A$, et, par suite, à l'intérieur de l'angle défini par les inégalités:

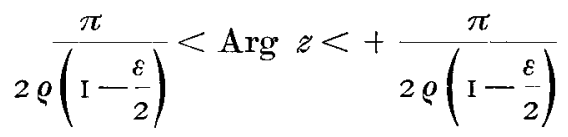

il existe un domaine (A), d'un seul tenant, en tout point duquel la fonction $f(z)$ vérifie l'inégalité:

$$
\log |f(z)|>\varepsilon|z|^{(1-\varepsilon)}
$$

Il résulte de cette propriété que la fonction $f(z)$ est à croissance régulière au sens de M. Borel: le maximum $V(r)$ de $\log |f(z)|$ sur le cercle $|z|=r$ vérifie les inégalités:

$$
r^{e[1-\varepsilon(r)]}<V(r)<r^{\varrho[1+\varepsilon(r)]}
$$

$\varepsilon(r)$ est une fonction tendant vers zéro lorsque $r$ tend vers l'infini.

La restriction fondamentale que nous avons faite sur la fonction $f(z)$ n'est pas nécessaire pour que cette conclusion soit valable: il suffit en effet que la fonction $\varphi(z)$ tende vers zéro sur les rayons $O C$ et $O C^{\prime}$, ce qui a lieu par exemple lorsque l'ordre de la fonction $f(z)$ sur ces rayons est inférieur à $\varrho(\mathrm{I}-2 \varepsilon)$. 
49. Le domaine $d$ des points où la fonction $f(z)$ satisfait à l'inégalité (3o) peut empiéter sur l'extérieur de l'angle $A^{\prime} O A$; mais, d'après l'hypothèse restrictive formulée sur la fonction $f(z)$, on pourra toujours supposer la constante $\varepsilon$ (du numéro 48) suffisamment petite pour qu'aucun argument limite des points de ce domaine ne soit supérieur en valeur absolue à $\frac{\pi}{\varrho}$.

L'application du théorème XI montre qu'il existe des points du domaine $(\mathbb{A})$ sur tout segment de la partie positive de l'axe réel, défini par les inégalités:

$$
\gamma^{.1-2 \varepsilon}<x<\vartheta^{1+2 \varepsilon}
$$

dès que $r$ dépasse une constante dépendant de $\varepsilon$.

Il résulte également de ce théorème qu'étant donnée une demi-droite quelconque d'un angle $B^{\prime} O B$ complètement intérieur à l'angle $A^{\prime} O A$, et une quantité $a$, on a l'inégalité:

$$
\max |f(z)-a|>\mathrm{I}
$$

sur tout segment de la demi-droite, défini par les inégalités:

$$
r^{1-\varepsilon_{1}(r)}<|z|<r^{.1+\varepsilon_{1}(r)}
$$

$\varepsilon_{1}(r)$ étant une fonction tendant vers zéro lorsque $r$ tend vers l'infini. Cette fonction est la même pour toutes les demi-droites intérieures à l'angle $B^{\prime} O B$.

50. Nous allons effectuer l'étude de la fonction $f(z)$ dans l'angle $A^{\prime} O A$ et montrer que l'exposant de convergence des zéros de $f(z)$ - a situés dans l'angle $B^{\prime} O B$ complètement intérieur à l'angle $A^{\prime} O A$ est inférieur à $\varrho$, quel que soit a.

Supposons que cette propriété ne soit pas vérifiée pour une certaine valeur $a$; quitte à remplacer la fonction $f(z)$ par la fonction $f(z)-a$, nous pourrons supposer qu'il s'agit des zéros de la fonction $f(z)$.

Choisissons un nombre positif $\alpha$ suffisamment petit pour que tous les cercles, dont les centres sont intérieurs à l'angle $B^{\prime} O B$, et dont les rapports deș rayons aux distances de leurs centres à l'origine sont égaux à I ooo $\alpha$, soient situés dans un angle complètement intérieur à l'angle $A^{\prime} O A$.

Ceci posé, il éxiste une suite de valeurs de $z$ tendant vers l'infini dans l'angle $B^{\prime} O B$, telles que les cercles $(C)$ de centre $z$ et de rayon $\alpha|z|$ contiennent un nombre de zéros de $f(z)$ supérieur à:

$$
|z|^{p\left[1-\varepsilon_{2}(|\hat{z}|)\right]}
$$

$\delta_{2}(|z|)$ étant une fonction tendant vers zéro lorsque $|z|$ augmente indéfiniment. 
En effet, s'il n'en était pas ainsi, en décomposant l'angle $B^{\prime} O B$ en circonférences de centres $z$ et de rayons $\alpha|z|$, de façon que tout point soit intérieur à l'une de ces circonférences, on constaterait que l'exposant de convergence des zéros de $f(z)$ situés dans l'angle $B^{\prime} O B$ serait inférieur à $\varrho$, ce qui est contraire à l'hypothèse.

Étudions la fonction $f(z)$ dans l'un des cercles $(C)$, le centre de ce cercle étant désigné par $z_{0}$.

51. Premier cas. - Dans le cercle $(C)$, on a l'inégalité:

$$
\log |f(z)|<-\frac{\mathrm{I}}{\mathrm{IOO}}\left|z_{0}\right| \rho\left[1-\varepsilon_{\underline{z}}\left(\left|z_{0}\right|\right)\right\}
$$

D'après l'une des propriétés précisées au numéro 49 on sait que la valeur de $|f(z)|$ est supérieure à un en un point $M$ dont l'argument est celui de $z_{0}$ et dont le module est compris entre:

$$
\left|z_{0}\right|^{1-\varepsilon_{1}\left(\left|z_{1}\right|\right)} \text { et }\left|z_{0}\right|^{1+\varepsilon_{1}\left(\left|z_{0}\right|\right)}
$$

Traçons, comme au numéro 40, un suite de circonférences ayant leurs centres sur le segment joignant $M$ au centre $z_{0}$ du cercle $(C)$, le rapport des rayons aux distancés de leurs centres à l'origine étant égal à $\alpha$; de plus, chaque circonférence a son centre situé sur la circonférence concentrique à la précédente et de rayon moitié.

En partant de la circonférence $(C)$, on aboutit nécessairement à une circonférence $\left(C_{1}\right)$ dans laquelle le maximum de $|f(z)|$ est supérieur à un, tandis que cette propriété n'a pas lieu pour les circonférences intermédiaires. En suivant la méthode exposée au numéro 40 , on constate que dans la circonférence $\left(C_{2}\right)$ concentrique à $\left(C_{1}\right)$ et de rayon moitié, la fonction $f(z)$ vérifie l'inégalité:

$$
\log |f(z)|<-\left.|z|\right|^{\left[1-z_{3}(|z|)\right]}
$$

$\varepsilon_{3}(|=|)$ est une fonction dépendant des fonctions $\varepsilon_{2}$ et $\varepsilon_{1}$; il est inutile de donner son expression exacte: il suffit de préciser que cette fonction tend vers zéro lorsque $|z|$. augmente indéfiniment, comme cela résulte du nombre des circonférences intermédiaires entre $(C)$ et $\left(C_{1}\right)$, et de l'application de l'inégalité de $\mathbf{M}$. Carleman dont il s'agit dans la note du numéro 40.

D'après le théorème XIII, le cercle concentrique au cercle $\left(C_{1}\right)$ et de rayon 30 fois plus grand, soit $\left(C_{1}^{\prime}\right)$, est un cercle de remplissage, et les propriétés de 
la fonction $f(z)$ dans ce cercle sont précisées dans ce théorème. On passe de là aisément, par application du théorème II, à un cercle de remplissage $\left(C_{3}\right)$ vu de l'origine sous un angle qui tend vers zéro lorsque la distance à l'origine du centre de ce cercle tend vers l'infini. On pent choisir le rayon de ce cercle de façon que le nombre des zéros de $f(z)-a$ soit (sauf pour des valeurs exceptionnelles de $a$ maintes fois précisées) supérieur à:

$$
|z|^{\left[1-\varepsilon_{\sharp}(|z|)\right]}
$$

$\varepsilon_{4}(|z|)$ étant une fonction qui tend vers zéro lorsque le module de l'affixe $z$ du centre du cercle $\left(C_{3}\right)$ tend vers l'infini.

D'après le choix de la constante $\alpha$, le cercle $\left(C_{3}\right)$ est intérieur à un angle complètement intérieur à l'angle $A^{\prime} O A$. On aboutit à la contradiction, puisque une suite de cercles $\left(C_{3}\right)$ s'éloignant indéfiniment, engendre une demi-droite de Borel intérieure à l'angle $A^{\prime} O A$.

52. Deuxième cas. - En un point intérieur au cercle $(C)$, et par suite sur une courbe $(\Gamma)$ issue de ce point, et s'éloignant à l'infini, la fonction $f(z)$ vérifie l'inégalité:

$$
\log |f(z)| \geq-\frac{\mathrm{I}}{\mathrm{IOO}}|z|^{\rho\left[1-\varepsilon_{\mathrm{g}}|z| \boldsymbol{|} \mid\right]}
$$

Nous allons montrer qu'un certain cercle, concentrique à $(C)$, est cercle de remplissage générateur d'une courbe de Borel.

Opérons la transformation conforme du cercle $(C)$ sur le cercle $|x|=1$, par similitude (pour simplifier l'écriture). En tout point $x_{1}$ de la courbe $(\gamma)$ transformée de la courbe $(I)$, de module compris entre $\mathrm{I}$ et 2 , on a l'inégalité:

$$
N\left(\left|x-x_{1}\right|=4,0\right)>\frac{I}{4}\left|z_{0}\right|^{\left.\left[1-\varepsilon_{2}|| z_{0} \mid\right]\right]}
$$

qui résulte du nombre des zéros de $g(x)$ (fonction transformée de $f(z)$ ) situés dans le cercle $|x|=\mathrm{I}$.

En tenant compte de la limitation inférieure de $\left|g\left(x_{1}\right)\right|$, due à ce que le point $x_{1}$ se trouve sur la courbe $(\gamma)$, et en appliquant la première inégalité fond̈amentale de M. R. Nevanlinna, on obtient l'inégalité:

$$
T\left(\left|x-x_{1}\right|=4, g\right)>\frac{\mathrm{I}}{8}\left|z_{0}\right|^{\left.\left[1-\varepsilon_{2}\left|z_{0}\right|\right)\right]}
$$


d'où résulte, d'après une propriété de la fonction caractéristique $T\left(v^{\prime}\right)$ d'une fonction holomorphe, l'inégalité:

$$
\underset{|x|=6}{\operatorname{Max}}|g(x)|>T\left(\left|x-x_{1}\right|=4, g\right)>\frac{1}{8}\left|z_{0}\right|^{\left[1-\varepsilon_{2}\left(\left|z_{0}\right|\right)\right]} .
$$

Cesi posé: ou bien il existe un cercle de centre $x=0$, et de rayon compris entre 6 et 7 , sur lequel le minimum de $|g(x)|$ est inférieur à $r o$, et alors, d'après un théorème classique, le nombre des zéros de $g(x)-a$ intérieurs à ce cercle est le même pour toute valeur de $a$ inférieure à ıo, ce qui entraîne, d'après le corollaire $d u$ théorème $I$, la conséquence suivante: le cercle correspondant, dans le plan des 2 , au cercle $|x|=140$ est un cercle de remplissage dans lequel le nombre des zéros de $f(z)-a$ est, sauf pour des valeurs exceptionnelles de $a$, de l'ordre de:

$$
\left|z_{0}\right|^{\left\lceil\left[1-\varepsilon_{2}\left(\left|z_{0}\right|\right)\right]\right.}
$$

On procède de nouveau à la division de ce cercle de remplissage en cercles plus petits, comme au numéro $5 \mathrm{I}$, de façon à avoir des cercles de remplissage vus de l'origine sous des angles qui tendent vers zéro; ces derniers cercles sont générateurs d'au moins une demidroite de Borel intérieure à l'angle $A^{\prime} O A$, ce qui est en contradiction avec l'hypothèse.

Ou bien sur tout cercle de centre $x=\mathrm{o}$ et de rayon compris entre 6 et 7 , la fonction $g(x)$ vérifie la condition:

$$
\min |g(x)| \leq \mathrm{IO} .
$$

On sait d'autre part que le maximum de $|g(x)|$ satisfait à l'inégalité (3 $\mathrm{I})$. Par. conséquent il existe, sur tout cercle de centre $x=0$ et de rayon compris entre 6 et 7 , un point $x_{1}$ où la fonction $g(x)$ et sa dérivée vérifient les inégalités:

$$
\begin{aligned}
& \left|g\left(x_{1}\right)\right|<\text { I I } \\
& \left|g^{\prime}\left(x_{1}\right)\right|>\text { I. }
\end{aligned}
$$

En appliquant la méthode fondamentale qui nous a conduit au théorème $I$; on constate aisément que, dans le cercle $|x|=40$, le nombre total des zéros de $g(x)$ et de $g(x)$ - I d'une part, le nombre total des zéros de $g(x)-2$ et de $g(x)-3$ d'autre part, sont tous deux supérieurs à:

$$
k\left|z_{0}\right|^{p\left[1-\varepsilon_{2}\left(\left|z_{0}\right|\right]\right)}
$$


$k$ désignant une constante numérique. D'après le corollaire du théorème $\mathrm{I}$, le nombre des zéros de $g(x)-a$ situés dans le cercle $|x|=800$ est supérieur à:

$$
\frac{n}{700}=\frac{k}{700}\left|z_{0}\right|^{p\left[1-\varepsilon_{2}\left(\left|z_{u}\right|\right)\right]}
$$

sauf pour des valeurs exceptionnelles de $a$ précisées dans ce corollaire.

On procède comme plus haut à la division du cercle correspondant, dans le plan des $z$, au cercle $|x|=800$, et, grâce à la valeur prise pour la constante positive $\alpha$, on prouve, de même que plus haut, l'existence d'une suite de cercles de remplissage générateurs d'une demidroite de Borel intérieure à l'angle $A^{\prime} O A$, ce qui est en contradiction avec l'hypothèse.

53. L'exposant de convergence des zéros de $f(z)$ - $a$ intérieurs à un angle $B^{\prime} O B$ complètement intérieur à l'angle $A^{\prime} O A$ est donc inférieur à $\varrho$ quel que soit $a$. Désignons par $a_{1}, a_{2}, \ldots a_{n}, \ldots$ les zéros de $f(z)$ situés dans l'angle $B^{\prime} O B$, et formons le produit canonique $\varphi(z)$, d'ordre $\varrho^{\prime}$ inférieur à $\varrho$, admettant les $a_{n}$ comme zéros. Nous nous proposons d'étudier la fonction $\frac{f(z)}{\varphi(z)}$ dans un angle $C^{\prime} O C$ complètement intérieur à l'angle $B^{\prime} O B$, et de montrer que, dans cet angle $C^{\prime} O C$, la fonction $\frac{f(z)}{\varphi(z)}$ converge uniformément vers l'infini, en satisfaisant à l'inégalité:

$$
\log \left|\frac{f(z)}{\varphi(z)}\right|>|z|^{\left.0\left[1-\varepsilon_{5}(1) \mid 1\right)\right]}
$$

$\varepsilon_{5}(|z|)$ étant une fonction qui tend vers zéro lorsque $|z|$ augmente indéfiniment. La fonction $\frac{f(z)}{\varphi(z)}$ est une fonction entière d'ordre $\varrho$, et dont l'ordre, sur toute demi-droite intérieure à l'angle $A O A^{\prime}$ (d'ouverture $\left.2 \pi-\frac{\pi}{\varrho}\right)$ est inférieur à un nombre fixe inférieur à $\varrho$, tout comme pour la fonction $f(z)$. La première propriété démontrée au numéro 49 pour la fonction $f(z)$ est donc aussi valable pour la fonction $\frac{f(z)}{\varphi(z)}$, c'est-à-dire qu'en un point an moins de tout segment de la partie positive de l'axe réel, défini par les inégalités:

$$
\gamma^{1-1-2 \varepsilon(r)}<x<r^{1+2 \varepsilon(r)}
$$

la fonction $\frac{f(z)}{\varphi(z)}$ satisfait à l'inégalité: 


$$
\log \left|\frac{f(z)}{\varphi(z)}\right|>\varepsilon(|z|)|z|^{[1-\varepsilon(|z|)]} .
$$

On peut choisir pour $\varepsilon(r)$ une fonction qui tend vers zéro lorsque $r$ tend vers l'infini, pas trop rapidement, de façon que le deuxième membre de l'inégalité précédente soit de la forme:

$$
|z|^{\left[1-\varepsilon_{1}|| z \mid\right]}
$$

$\varepsilon_{1}(|z|)$ tendant vers zéro avec $\frac{\mathrm{I}}{|z|}$.

Ceci posé, soient $O D$ et $O D^{\prime}$ les bissectrices intérieures des angles $C O B$ et $B^{\prime} O C^{\prime}$; la fonction $\frac{f(z)}{\varphi(z)}$ est supérieure en module à un dans l'angle $D^{\prime} O D$, powrvu que $|z|$ soit assez grand. Supposons en effet qu'il n'en soit pas ainsi, c'està-dire que la fonction considérée est inférieure on égale à un en module, en une suite de points tendant vers l'infini à l'intérieur de l'angle $D^{\prime} O D$.

En joignant, par une suite de cercles, chacun de ces points au point le plus proche en lequel l'inégalité (33) est vérifiée, on prouve l'existence d'un cercle de remplissage $(c)$ dans lequel le maximum et le minimum de $\log \left|\frac{f(z)}{\varphi(z)}\right|$ sont respectivement de la forme: $|z|^{\rho(1-\varepsilon)}$ et $-|z|^{\rho(1-\varepsilon)}$; on en déduit que sur une courbe traversant la couronne circulaire comprise entre $(c)$ et le cercle concentrique et de rayon double, la fonction $\frac{f(z)}{\varphi(z)}$ est inférieure en module à son minimum dans le cercle (c), puisqu'elle est dépourvue de zéros dans cette région du plan. Il en résulte pour la fonction $f(z)$ une inégalité de la forme:

$$
\log |f(z)|<-|z|^{[[1-\varepsilon(|z|)]}
$$

sur cette même courbe. En remarquant que le maximum de $\log |f(z)|$ est très grand dans le cercle $(c)$, on en déduit, d'après le théorème XIII, l'existence d'un cercle de remplissage, pour la fonction $f(z)$, dans les environs du cercle (c); et, en considérant une suite de cercles $(c)$, s'éloignant à l'infini dans l'angle $D^{\prime} O D$, il en résulte l'existence d'une demi-droite de Borel complètement intérieure à l'angle $A^{\prime} O A$, ce qui est contraire à l'hypothèse.

Done, dès que $|z|$ est assez grand, le module de la fonction $\frac{f(z)}{\varphi(z)}$ est supérieur à un à l'intérieur de l'angle $D^{\prime} O D$, et, en une suite de points situés sur $32-2822$. Acta mathematica. 52. Imprimé lo 19 septembre 1928. 
la partie positive de l'axe réel, il satisfait à l'inégalité (33). En rayonnant à partir de ces points, et appliquant à la fonction $\frac{\varphi(z)}{f(z)}$ la proposition rappelée dans la note du numéro 40, dans des cercles intérieurs à l'angle $D^{\prime} O D$, on constate qu'en tout point $z$ situé dans l'angle $C^{\prime} O C$, qui est complètement intérieur à l'angle $D^{\prime} O D$, la fonction $\frac{f(z)}{g(z)}$ vérifie l'inégalité (32) recherchée.

54. Etudions la fonction $f(z)$ à l'intérieur de l'angle $A O A^{\prime}$, dont l'ouverture, égale à $2 \pi-\frac{\pi}{\varrho}$, est supérieure à̀ $\frac{\pi}{\varrho}$.

Dans tout angle complètement intérieur à l'angle $A O A^{\prime}$, l'exposant de convergence des zéros de $f(z)-a$ ne peut être, pour aucune valeur de $a$, égal à $\varrho$. En effet, s'il était égal à $\varrho$, il serait possible de construire, à l'intérieur de l'angle $A O A^{\prime}$, un angle d'ouverture supérieure à $\frac{\pi}{\varrho}$, englobant ces zéros; d'après un théorème de $M$. R. Nevanlinna, l'ordre de la fonction $f(z)$ dans cet angle, serait égal à $\varrho$, ce qui est contraire à l'hypothèse.

55. Donc toute fonction $f(z)-a$ peut être décomposée en un produit de la forme:

$$
f(z)-a=\varphi(z) \psi(z)
$$

$\varphi(z)$ étant une fonction entière (produit canonique par exemple) d'ordre inférieur $\grave{a} \varrho$, et $\psi(z)$ une fonction entière d'ordre $\varrho$, dont les zéros ont pour seuls arguments limites $\pm \frac{\pi}{2 \varrho}$. Cette fonction $\psi(z)$ est à croissance régulière et converge uniformément vers l'infini dans tout angle complètement intérieur à l'angle $A^{\prime} O A$.

Nous allons montrer que l'exposant de convergence des zéros de $f(z)-a$, ayant pour argument limite soit $+\frac{\pi}{2 \varrho}$, soit $-\frac{\pi}{2 \varrho}$, est nécessairement égal à @.

Supposons qu'il n'en soit pas ainsi, pour $a=0$, pour fixer les idées, et pour la demi-droite $O A$ d'argument $+\frac{\pi}{2 \varrho}$. On pourra supposer que les zéros de $\psi(z)$ ayant pour argument limite $+\frac{\pi}{2 \varphi}$ ont été incorporés à la fonction $\varphi(z)$, de sorte que $\psi(z)$ est une fonction entière d'ordre $\varrho$ dont les zéros ont pour seul argument limite $-\frac{\pi}{2 \varrho}$. 
Dans l'un quelconque (e) des cercles de remplissage dont les points ont des arguments voisins de $\frac{\pi}{2 \varrho}$, le nombre des zéros de $f(z)-a$ est, sauf pour $a$ très voisin de $o$, ou pour $a$ très grand, supérieur à

$$
r^{2}(1-\varepsilon)
$$

$v$ désignant le module de l'affixe du centre du cercle de remplissage et $\varepsilon$ une fonction de $r$ qui tend vers zéro lorsque $r$ augmente indéfiniment; le nombre des zéros de $f(z)$ dans ce même cercle (et même dans un cercle $\left(c^{\prime}\right)$ concentrique et de rayon double) est inférieur à $r^{Q^{\prime}}, \varrho^{\prime}$ étant une constante inférieure à $\varrho$, indépendante du cercle de remplissage choisi.

Il résulte alors du théorème IX qu'en tout point $z$ d'une courbe $(T)$ issue d'un point intérieur au cercle $\left(c^{\prime}\right)$ et traversant une certaine couronne circulaire, la fonction $f(z)$ vérifie l'inégalité:

$$
\log |f(z)|<-|z|^{o\left(1-\varepsilon^{\prime \prime}\right)}
$$

$\varepsilon^{\prime \prime}$ étant une fonction de $|z|$ qui tend vers zéro lorsque $|z|$ augmente indéfiniment.

En isolant les zéros de la fonction $\varphi(z)$ à l'intérieur de couronnes circulaires; de façon à appliquer le théorème de $\mathbf{M}$. Hadamard sur le minimum du module de $\varphi(z)$ à l'extérieur de ces couronnes, on constate qu'il existe nécessairement des points de la courbe $(\Gamma)$ en lesquels la fonction $\psi(z)=\frac{f(z)}{\varphi(z)}$ vérifie une inégalité analogue à l'inégalité précédente. Comme la fonction $\psi(z)$ n'a pas de zéros extérieurs à un angle, qu'on peut prendre d'ouverture arbitrairement petite, ayant pour bissectrice la demi-droite d'argument $-\frac{\pi}{2 \varrho}$ il en résulte que la fonction $\psi(z)$ vérifie une inégalité de la forme:

$$
\log |\psi(z)|<-|z|^{\rho\left(1-\varepsilon^{\prime}\right)}
$$

non seulement en un point de la courbe $(\Gamma)$, mais aussi sur une courbe $\left(\Gamma^{\prime}\right)$ issue de ce point.

La fonction $\psi(z)$ converge uniformément vers l'infini dans tout angle complètement intérieur à l'angle $A^{\prime} O A$. Donc: ou bien la courbe $\left(I^{\prime}\right)$ empiète sur un angle complètement intérieur à l'angle $A O A^{\prime}$, ou bien tous ses points ont des arguments voisins de $\frac{\pi}{2 \varrho}$. 
Cette dernière hypothèse est en contradiction avec l'application du théorème XI (voir aussi la remarque faite au numéro $3 \mathrm{I}$ ) à l'étude de la fonction $\frac{\mathrm{I}}{\psi(z)}$ à l'extérieur d'un angle comprenant la demi-droite d'argument $-\frac{\pi}{2 \varrho}$ : cette fonction $\frac{\mathrm{I}}{\psi(z)}$ y est holomorphe et d'ordre $\varrho$, et les arguments des points où la fonction $\frac{1}{\psi(z)}$ est d'ordre $\varrho$ sont compris entre des valeurs qui diffèrent au moins de $\frac{\pi}{\varrho}-\varepsilon_{1}$.

Quant à la première hypothèse, elle entraîne nécessairement l'existence, dans un secteur de couronne circulaire complètement intérieur à l'angle $A O A^{\prime}$, d'ouverture supérieure à $\frac{\pi}{\varrho}$, et coupant la courbe $\left(I^{\prime}\right)$, d'un cercle de remplissage (voir le théorème XVI) non seulement pour la fonction $\frac{\mathrm{I}}{\psi(z)}$, mais, par répercussion, pour la fonction $\psi(z)$. Dans un tel cercle, le maximum. de $\psi(z)$ est de la forme $|z|^{\left(1-\varepsilon_{2}\right)}$; il en est de même du maximum de $|f(z)|$, ce qui est en contradiction avec l'ordre de la fonction $f(z)$ à l'intérieur de l'angle $A O A^{\prime}$.

Remarquons que la démonstration précédente s'étend immédiatement au cas où la fonction $\psi(z)$, au lieu d'être privée de zéros aux environs de toute la demidroite $O A$, serait seulement dépourvue de zéros dans les environs de cette demidroite situés à l'interieur de la couronne circulaire:

$$
r^{1-\gamma}<|z|<r^{1+\gamma}
$$

$(\gamma$ est une constante positive).

Il suffit, dans ce cas, d'effectuer l'étude des fonctions $f(z), \psi(z)$ et $\frac{\mathrm{I}}{\psi(z)}$ dans des secteurs de couronnes circulaires, et d'appliquer, comme nous l'avons fait plus haut, les résultats des théorèmes XI et XVI.

Il en résulte que le nombre des zéros de $f(z)$-a ne peut être inférieur à $r^{(1-\varepsilon)}$ dans tout sectewr de couronne circulaive défini par les inégalités:

$$
\begin{gathered}
r^{1-\gamma}<|z|<r^{1+\gamma} \\
\frac{\pi}{\varrho}-\eta<|\operatorname{Arg} z|<\frac{\pi}{\varrho}+\eta
\end{gathered}
$$


$\varepsilon, \gamma$ et $\eta$ sont des quantités positives, qu'on peut prendre constantes, ou fonctions de $r$ tendant vers zéro lorsque $r$ augmente indéfiniment. ${ }^{1}$

56. Résumons les plus marquants des résultats précédents:

XVIII. Soit $f(z)$. une fonction entière, d'ordre fini $\varrho$ supérieur à un, ne pos. sédant que deux demi-droites de Borel $O A^{\prime}$ et $O A$.

L'un des angles de ces demi-droites, soit $A^{\prime} O A$, est nécessairement égal à $\frac{\pi}{\varrho}$.

En outre, si la fonction $f(z)$ est d'ordre inférieur à un nombre fixe inférieur. $\grave{a} \varrho$ dans tout angle complètement intérieur à l'angle $A O A^{\prime}$, l'exposant de convergence des zéros de $f(z)-a$ est, quel que soit a, égal à $\varrho$ dans tout angle contenant l'une quelconque des demidroites de Borel, et inférieur $\grave{a} \varrho$ dans tout angle complètement interieur à l'un quelconque des angles $A^{\prime} O A, A O A^{\prime}$.

De plus, soit $\varphi(z)$ le produit canonique (d'ordre infëriew à $\varrho$ ) dont les zéros sont ceux de $f(z)$ qui sont contenus dans un angle $B^{\prime} O B$ complètement intérieur $\grave{a}$ l'angle $A^{\prime} O A$. Dans tout angle complètement intérieur à l'angle $B^{\prime} O B$, on a l'inégalité:

$$
\log \left|\frac{f^{f}(z)}{\varphi(z)}\right|>|z|^{[1-\varepsilon(\mathbf{I} z \mid)]}
$$

$\varepsilon(|z|)$ est une fonction qui tend vers zéro lorsque $|z|$ augmente indéfiniment.

Une partie des résultats contenus dans ce théorème répond à une question posée par M. Valiron dans son récent mémoire des Acta (Recherches sur le théorème de $M$. Borel . ., cité plus haut; voir p. 92).

57. Comme nous l'avons $\mathrm{vu}$ au numéro 47 , la restriction formulée pour les fonctions entières n'admettant que deux demi-droites de Borel n'a plus sa raison d'être dans le cas d'une fonction entière n'admettant que deux demi-droites de Julia.

Dans ce cas, en plus des propriétés précisées dans le théorème XVIII, la fonction converge uniformément vers l'infini dans tout angle $B^{\prime} O B$ complètement intérieur à l'angle $A^{\prime} O A$.

Cette propriété, particulière aux fonctions entières ne possédant que deux

1 On peut également démontrer que l'exposant de convergence des zéros de $f(z)-a$ ayant pour argument limite l'une des valeurs $\pm \frac{\pi}{2 \varrho}$ est égal à $\varrho$, en étudiant les propriétés de la fonction entière $\psi(z)$, d'ordre $\varrho$, dont les zéros ont un seul argument limite, et en montrant que, puisqn'il existe un angle $A^{\prime} O A$, d'ouverture $\frac{\pi}{\rho}$, situé d'un côté de la demi-droite $O A^{\prime}$ dont l'argument est l'argument limite des zéros de $\psi(z)$, et tel que sur toute demi-droite intérieure à l'angle $A^{\prime} O A$ la fonction $\psi(z)$ est d'ordre $\varrho$, cet angle se reproduit par symétrie par rapport à $O A^{\prime}$, ce qui est contradictoire avec les propriétés de la fonction $f(z)$ dans l'angle $A O A^{\prime}$. 
demi-droites de Julia, résulte de ce fait que s'il existe une suite de points intérieurs à l'angle $B^{\prime} O B$, en lesquels le module de la fonction est inférieur à un, en les joignant aux points les plus proches vérifiant l'inégalité (32), on établit l'existence d'une suite de cercles de remplissage situés dans un angle complètement intérieur à l'angle $A^{\prime} O A$, ce qui est contradictoire avec la définition de la fonction considérée.

On passe de là à la démonstration de l'inégalité:

$$
\log |f(z)|>|z|^{\rho[1-\varepsilon(\{z \mid)]}
$$

(le point $z$ étant situé dans un angle complètement intérieur à l'angle $B^{\prime} O B$ ), comme nous l'avons fait pour la fonction $\frac{f(z)}{\varphi(z)}$ au $n^{\circ} 53 .^{1}$

58. Les fonctions entières de Mittag-Leffler et de M. Lindelöf ${ }^{2}$ sont des fonctions ne possédant que deux demi-droites de Julia. Par exemple la fonction entière de $M$. Lindelöf:

$$
f(z)=\sum_{n=1}^{\infty}\left(\frac{z}{n^{\frac{1}{q}}}\right)^{n}
$$

converge uniformément vers l'infini dans tout angle complètement intérieur à l'angle $A^{\prime} O A$, d'ouverture $\frac{\pi}{\varrho}$, déterminé par les droites $O A$ et $O A^{\prime}$ d'arguments $\pm \frac{\pi}{2 Q}$, et converge uniformément vers zéro dans tout angle complètement intérieur à intérieur à l'angle $A O A^{\prime}$.

La fonction de Mittag-Leffler:

$$
f(z)=\sum_{n=0}^{\infty} \frac{z^{n}}{\Gamma\left(\mathrm{I}+\frac{n}{\varrho}\right)}
$$

jouit de propriétés analogues.

On peut former d'autre exemples de fonctions entières n'admettant que deux demi-droites de Julia en ajoutant aux fonctions précédentes (ou en multipliant ces fonctions par) une fonction entière d'ordre inférieur à $\frac{I}{2}$, dont les zéros ont

1 M: G. Valiron a établi une démonstration analogue dans une note des Compte-Rendus (Sur quelques propriétés des fonctions entières, t. 185, 1927, p. 1439).

${ }^{2}$ Lindelöf. Calcul des résidus, p. II9. 
pour seul argument limite l'une des valeurs $\pm \frac{\pi}{2 \varrho}$, et satisfaisant à la propriété suivante: cette fonction converge uniformément vers l'infini à l'extérieur de cercles entourant ses zéros, et les rapports des rayons de ces cercles aux distances de leurs centres à l'origine tendent vers zéro lorsque ces cercles s'éloignent indéfiniment (on sait qu'il existe de telles fonctions entières).

On obtiendra une fonction entière ne possédant que deux demi-droites de Borel en multipliant une fonction ne possédant que deux demi-droites de Julia (par exemple la fonction de $\mathbf{M}$. Lindelöf) par une fonction entière quelconque d'ordre inférieur à $\varrho$. Ce produit satisfait à la condition restrictive énoncée au numéro 46.

59. Terminons ce mémoire en donnant quelques indications sur certaines fonctions entières d'ordre infini: le théorème $\mathrm{XV}$ s'applique à une telle fonction, et l'on peut donner à $\varrho^{\prime}$ une valeur tendant vers l'infini avec $r$; mais le théorème $\mathrm{XI}$ ne s'applique pas toujours comme nous l'avons remarqué au numéro 32 . S'il s'applique, on pourra choisir aussi pour $\varrho^{\prime}$ une valeur tendant vers l'infini avec $r$. On prouve ainsi, comme à la fin du chapitre IV pour les fonctions d'ordre fini, l'existence de deux suites de cercles de remplissage, s'éloignant à l'infini, et dans chacun desquels le nombre des zéros de $f(z)-a$ est, sauf pour des valeurs exceptionnelles de $a$, supérieur à $r{ }^{\prime \prime}, \varrho^{\prime \prime}$ étant une fonction de $r$ qui tend vers l'infini avec $r$. Les arguments limites de ces deux suites ne sont pas nécessairement différents, comme dans le cas des fonctions entières d'ordre fini. La différence des arguments de deux cercles de chacune des suites, dont les positions sont précisées au théorème $\mathrm{XV}$, peut tendre vers zéro comme $\frac{\pi}{\varrho^{\prime \prime}}$, lorsque $r$ augmente indéfiniment. 